\title{
Amino Acid Substitutions of Coiled-Coil Protein Tpr Abrogate Anchorage to the Nuclear Pore Complex but Not Parallel, In-Register Homodimerization
}

\author{
Manuela E. Hase, Nikolai V. Kuznetsov, and Volker C. Cordes* \\ Department of Cell and Molecular Biology, Medical Nobel Institute, Karolinska Institutet, S-17177 \\ Stockholm, Sweden
}

Submitted December 27, 2000; Revised April 9, 2001; Accepted May 30, 2001

Monitoring Editor: Pamela A. Silver

\begin{abstract}
Tpr is a protein component of nuclear pore complex (NPC)-attached intranuclear filaments. Secondary structure predictions suggest a bipartite structure, with a large N-terminal domain dominated by heptad repeats (HRs) typical for coiled-coil-forming proteins. Proposed functions for Tpr have included roles as a homo- or heteropolymeric architectural element of the nuclear interior. To gain insight into Tpr's ultrastructural properties, we have studied recombinant Tpr segments by circular dichroism spectroscopy, chemical cross-linking, and rotary shadowing electron microscopy. We show that polypeptides of the $\mathrm{N}$-terminal domain homodimerize in vitro and represent $\alpha$-helical molecules of extended rod-like shape. With the use of a yeast two-hybrid approach, arrangement of the coiled-coil is found to be in parallel and in register. To clarify whether Tpr can self-assemble further into homopolymeric filaments, the full-length protein and deletion mutants were overexpressed in human cells and then analyzed by confocal immunofluorescence microscopy, cell fractionation, and immuno-electron microscopy. Surplus Tpr, which does not bind to the NPC, remains in a soluble state of $\sim 7.5 \mathrm{~S}$ and occasionally forms aggregates of entangled molecules but neither self-assembles into extended linear filaments nor stably binds to other intranuclear structures. Binding to the NPC is shown to depend on the integrity of individual HRs; amino acid substitutions within these HRs abrogate NPC binding and render the protein soluble but do not abolish Tpr's general ability to homodimerize. Possible contributions of Tpr to the structural organization of the nuclear periphery in somatic cells are discussed.
\end{abstract}

\section{INTRODUCTION}

The nuclear pore complex (NPC) is a highly complex structure of eightfold rotational symmetry that serves as the gateway for the exchange of cellular material between cytoplasm and nucleus in eukaryotes. Its core structure consists of central globular subunits flanked by a ring-like structure (annulus) at both the NPC's cytoplasmic (outer) and nucleoplasmic (inner) side. Both annuli are attachment sites for

* Corresponding author. E-mail address: volker.cordes@cmb.ki.se. Abbreviations used: ATCC, American Type Culture Collection; aa, amino acid(s); $\mathrm{AD}$, activation domain; $\mathrm{BD}$, binding domain; BSA, bovine serum albumin; CD, circular dichroism; CMV, cytomegalovirus; EM, electron microscopy; FA, formaldehyde; GA, glutaraldehyde; GST, glutathione S-transferase; HR, heptad repeat; IF, immunofluorescence microscopy; Ig, immunoglobulin; $\mathrm{mAb}$, monoclonal antibody; $\beta$-ME, $\beta$-mercaptoethanol; NLS, nuclear localization signal; NPC, nuclear pore complex; PBS, phosphate-buffered saline; sulfo-EGS, ethyleneglycol bis[sulfosuccinimidylsuccinate]; X-Gal, 5-bromo-4-chloro-3-indolyl-Dgalactoside. fibrils, also arranged in an eightfold symmetrical pattern but of distinctive shape and protein composition (for recent reviews, see Ohno et al., 1998; Stoffler et al., 1999; Ryan and Wente, 2000). Fibrils emanating from the outer annulus exist as short tufts of $\sim 50 \mathrm{~nm}$ in length, whereas the rectilinear fibrils of $5-7 \mathrm{~nm}$ in diameter attached to the inner annulus vary in length. Approximately 60-100 nm from the NPC's inner annulus, the intranuclear fibrils are laterally interconnected by another ring-like structure, sometimes described as the "terminal ring." Together, terminal ring and fibrils proximal to the NPC proper are considered to represent a structural and functional entity, called the nuclear "fishtrap" or "basket" (Ris, 1989, 1991; Jarnik and Aebi, 1991; Goldberg and Allen, 1992).

Emanating from the terminal ring, additional fibrils project further into the nuclear interior. In amphibian oocytes these fibrils can be several hundred nanometers in length and appear to connect the NPCs with the cortex of amplified nucleoli (Franke and Scheer, 1970; Scheer et al., 1988; Cordes et al., 1993; Ris and Malecki, 1993; Arlucea et al., 1998). Of unknown function, they have been proposed to be 
involved in nucleocytoplasmic or intranuclear transport, or structural organization of the nucleus (Franke and Scheer, 1970; Scheer et al., 1988; Cordes et al., 1997; Gant and Wilson, 1997; Ris, 1997; Singer and Green, 1997; Pemberton et al., 1998; Herrmann and Lichter, 1999; Laskey, 2000).

In vertebrates, a $267-\mathrm{kDa}$ protein termed Tpr (translocated promotor region) has been localized to this filamentous material in both germ and somatic cells (Cordes et al., 1997). Originally, the tpr gene had been identified by its rearrangement in various tumor cell lines (Park et al., 1986; Ishikawa et al., 1987; Soman et al., 1991; Greco et al., 1992; Rodrigues and Park, 1993), resulting in fusions between short Tpr segments and proto-oncogenic kinases.

Homologues of Tpr have been identified in both Drosophila melanogaster and Saccharomyces cerevisiae. Whereas the majority of Tpr in mammalian cells appears to be located near the NPCs, Drosophila Tpr is found attached to NPCs and throughout the extrachromosomal and extranucleolar spaces of the nuclear interior (Zimowska et al., 1997). In yeast, two homologues of Tpr exist, Mlp1 and Mlp2, which are also located at the NPCs and deep within the nucleus (Strambio-de-Castilia et al., 1999; Kosova et al., 2000). However, it is unknown whether these intranuclear pools of Tpr are part of any higher order nuclear structures.

Predictions of secondary structure suggest that Tpr and its homologues are divided into two major domains (Mitchell and Cooper, 1992; Byrd et al., 1994; Zimowska et al., 1997; Strambio-de-Castillia et al., 1999): The smaller, C-terminal domain contains the nuclear localization signal (NLS; Cordes et al., 1998; Strambio-de-Castillia et al., 1999; Kosova et al., 2000) and does not appear to be dominated by a particular type of secondary structure. In contrast, the $\mathrm{N}$ terminal domain of $\sim 190 \mathrm{kDa}$ in humans, is predicted to be mainly composed of $\alpha$-helices and has numerous clusters of consecutive copies of heptad repeats (HRs). This sequence motif is characteristic for many $\alpha$-helical proteins organized in homo- or heteromeric coiled-coils (Lupas, 1996). Different segments of the domain's first, but not second, half have been shown to bind to NPCs (Bangs et al., 1998; Cordes et al., 1998). However, the sequence elements involved in and essential for NPC binding have not been characterized.

The function of Tpr and its insect and yeast homologues remains enigmatic. Yeast cells in which both MLP1 and MLP2 have been deleted are viable and exhibit only minor or no alterations in nucleocytoplasmic transport (Strambiode-Castillia et al., 1999; Galy et al., 2000; Kosova et al., 2000). Conversely, overexpression of Tpr in mammalian cells, and of Mlp1 in yeast, has been reported to cause intranuclear mRNA accumulation in a subpopulation of cells (Bangs et al., 1998; Kosova et al., 2000), but it remains unclear whether this reflects a direct role in nuclear transport (Paddy, 1998). Loss of the MLP2 gene product resulted in DNA repair deficiencies and disruption of perinuclear telomere clustering (Galy et al., 2000).

Proposed functions for Tpr and Mlp proteins include i) a contribution to NPC architecture, ii) direct roles in nucleocytoplasmic transport, iii) the formation of extensive filamentous tracks for intranuclear transport toward and away from the NPCs, iv) a role as a general architectural element of a fibrillar "nucleoskeleton," or v) participation in nuclear subdomain organization (Cordes et al., 1997; Gant and Wilson, 1997; Singer and Green, 1997; Zimowska et al., 1997;
Bangs et al., 1998; Paddy, 1998; Pemberton et al., 1998; Shah et al., 1998; Herrmann and Lichter, 1999; Strambio-de-Castillia et al., 1999; Galy et al., 2000; Kosova et al., 2000; Laskey, 2000).

Filament association, localization deep within the nucleus in some species, and the predicted coiled-coil structure of Tpr have led to speculations that this protein may be capable of self-assembling into stable homopolymeric filaments of considerable length. However, supporting experimental evidence is limited to an investigation in which N-terminal domain segments of human Tpr, expressed in a hamster kidney cell line, were reported to form long filaments extending throughout the cytoplasm (Bangs et al., 1998). However, whether these represented homopolymers of Tpr molecules, or rather an association of the recombinant Tpr to intrinsic cytoskeletal elements, remained elusive.

To follow up ideas that Tpr may serve as an architectural element within the nucleus, we have focused in this study on the following objectives: i) to gain insight into Tpr's basic secondary and quaternary structural properties and determine whether Tpr is a homo- or heteromeric coiled-coil protein, ii) to decide whether Tpr may form stable homopolymeric filaments, and iii) to determine whether any particular HRs may be involved in stably tethering the protein to the NPC or other structures.

\section{MATERIALS AND METHODS}

\section{Antibodies}

Mouse monoclonal antibody (mAb) 9E10 against the peptide sequence EQKLISEEDL of human c-myc protein (American Type Culture Collection [ATCC] CRL 1729), affinity-purified guinea pig and rabbit antibodies against amino acid (aa) 1622-1640 and aa 2063-2084 of human Tpr, and mAb 203-37 against Tpr's N-terminal domain (Cordes et al., 1997) have been described; in the present study, the epitope recognized by mAb 203-37 was mapped to locate between aa 1370 and 1623 of hTpr. MAbs against Gal4-BD and influenza virus HA epitope, used for immunodetection of Gal4-Tpr fusion proteins, were from Clontech (Palo Alto, CA) and Roche Molecular Biochemicals (Mannheim, Germany), respectively.

\section{cDNA Cloning and In Vitro Mutagenesis}

cDNAs encoding hTpr and polymerase chain reaction products encoding the C-terminal domain of Xenopus laevis Tpr have been described (Cordes et al., 1997, 1998). Subclonings were into i) mammalian expression vector pRC/CMV (InVitrogen, San Diego, CA), ii) bacterial expression vectors pGEX6P3 (Pharmacia, Uppsala, Sweden) and pQE30 (Qiagen, Hilden, Germany), and iii) yeast twohybrid expression vectors pAS2-1 and pACT-2 (Clontech). In vitro mutagenesis by polymerase chain reaction was according to the QuikChange site-directed mutagenesis method (Stratagene, La Jolla, CA). Propagation of Tpr-encoding constructs was usually in strain Escherichia coli Sure to avoid tpr recombination events observed in other strains.

\section{Expression Vector Constructs}

pRC/CMV constructs hTpr, myc.hTpr, myc.hTpr.1-1832(ANLS), myc.hTpr.1-1640 (ANLS), myc.hTpr.rod/SV40-NLS, myc.hTpr.1775/SV40-NLS, myc.hTpr.1-513/SV40-NLS, and myc.hTpr.7741653/SV40-NLS have been described (Cordes et al., 1998). Novel pRC/CMV constructs are outlined in Table 1. Yeast two-hybrid constructs encoding fusions between Tpr polypeptides, and $\mathrm{N}$ terminal Gal4-BD (pAS2-1) or Gal4-AD (pACT-2), are listed in Table 2 ; aa sequences are deduced from open reading frames downstream 
Table 1. Mammalian expression vector constructs

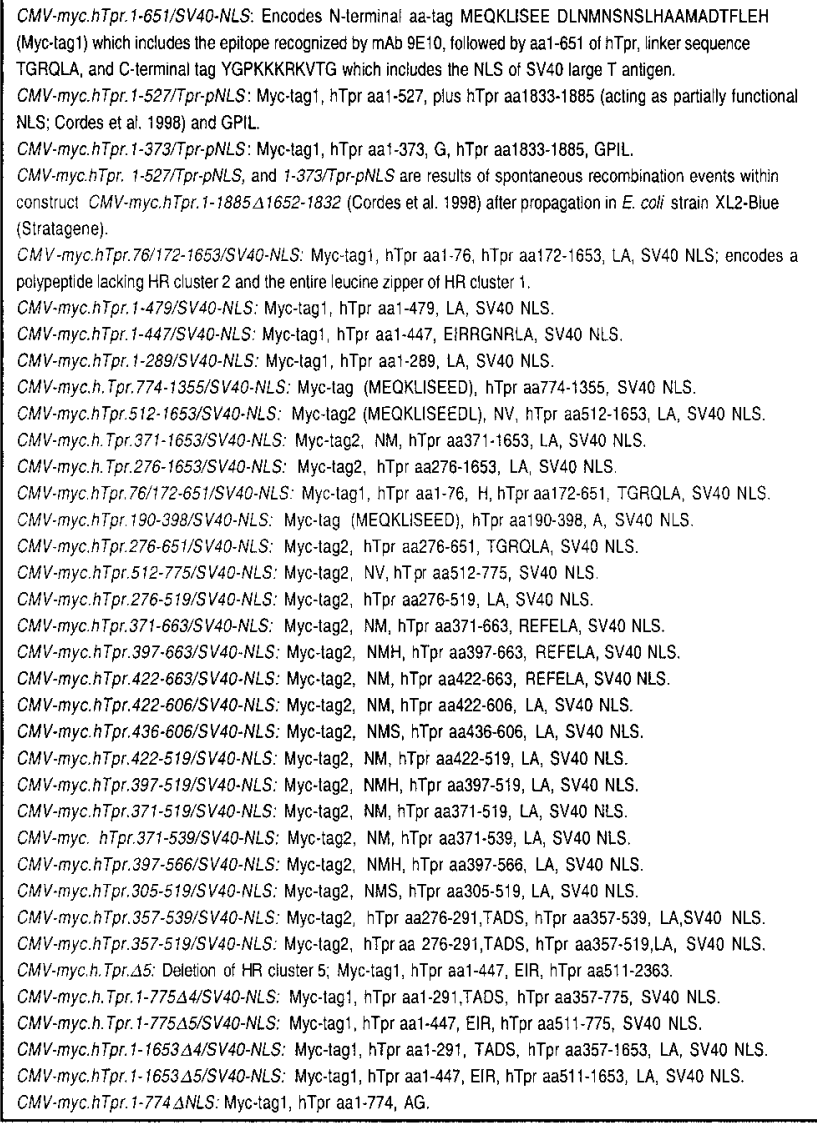

of the NcoI site in the vectors' MCS. Two-hybrid construct pASvimentin encodes a Gal4-BD fusion with full-length human vimentin; pLAM5'-1 (Clontech) is a pAS2-1 derivative encoding aa 66-230 of human lamin C. Bacterial expression vector constructs are described in Table 3.

\section{Bacterial Expression and Purification of Tpr Polypeptides}

Glutathione $S$-transferase (GST)-tagged polypeptides were synthesized in E. coli BL21-LysS. Cells were lysed by sonication in $50 \mathrm{mM}$ sodium phosphate, pH 7.8, with $300 \mathrm{mM} \mathrm{NaCl}$, and cleared lysates were incubated with glutathione Sepharose 4B (Pharmacia). The slurry was washed with $50 \mathrm{mM}$ sodium phosphate, pH 7.8, with 300 $\mathrm{mM} \mathrm{NaCl}$ and $0.04 \%$ Triton-X100, and bound proteins were eluted with $10 \mathrm{mM}$ reduced glutathione in $50 \mathrm{mM}$ Tris- $\mathrm{HCl}$, pH 8.0. GST tags were proteolytically removed with PreScission Protease (Pharmacia); tag-free polypeptides were stored in $50 \mathrm{mM}$ Tris- $\mathrm{HCl}, \mathrm{pH}$ 7.0, with $150 \mathrm{mM} \mathrm{NaCl}, 1 \mathrm{mM}$ EDTA, and $1 \mathrm{mM}$ dithiothreitol. His-tagged proteins were synthesized in E. coli M15[pREP4] (Qiagen) and cells were lysed by sonication in $50 \mathrm{mM}$ sodium phosphate, pH 7.5, with $150 \mathrm{mM} \mathrm{NaCl}$ and $10 \mathrm{mM}$ imidazole (Ni-buffer 1). Cleared lysates, supplemented with $2.5 \mathrm{mM} \beta$-mercaptoethanol $(\beta$-ME) and $4 \%$ glycerol (Ni-buffer 2$)$, were incubated with $\mathrm{Ni}$ nitrilotriacetic acid agarose (Qiagen, Chatsworth, CA), which was then washed with Ni-buffer 2 containing $40 \mathrm{mM}$ imidazole. Step-
Table 2. Yeast two-hybrid expression vector constructs

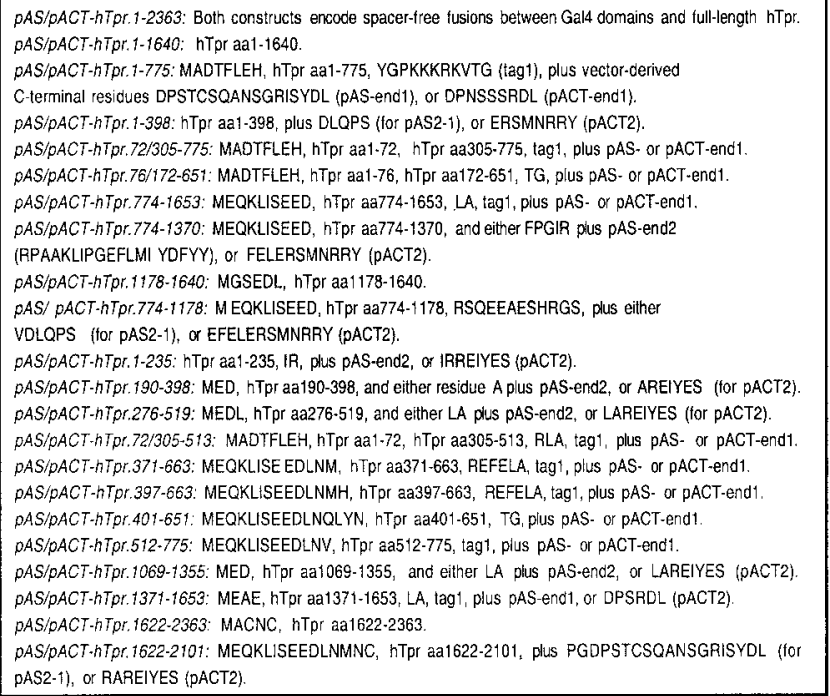

wise elutions of bound protein were in Ni-buffer 2 containing 75 , 100, 125, 150, 175, 200, 225, 250, and $500 \mathrm{mM}$ imidazole.

\section{Determination of Protein Concentration}

Approximate concentrations of N-terminal Tpr polypeptides in aqueous solutions were determined with the Bio-Rad Protein Assay (Bio-Rad Laboratories, Hercules, CA). Concentrations of C-terminal domain segments were determined by a modified Lowry protein assay (Pierce, Rockford, IL). For evaluation of circular dichroism (CD) spectra of N-terminal polypeptides, concentrations were determined by protein hydrolysis and ninhydrin-type aa analysis at the Protein Analysis Center at Karolinska Institutet (Stockholm, Sweden).

\section{Spectroscopy}

Polypeptides were dialyzed against $40 \mathrm{mM}$ sodium phosphate, $\mathrm{pH}$ 7.4 , and spectra were recorded with a Jasco J-720 spectropolarimeter (Japan Spectroscopic, Tokyo, Japan) at $15,20,25$, and $36^{\circ} \mathrm{C}$, with the use of cuvettes of 1- or $0.05-\mathrm{mm}$ pathlength. Wavelength range was from 250 to $190 \mathrm{~nm}$. Evaluation of CD spectra for secondary structure predictions was with the use of a modified version (provided by Dr. Jüri Jarvet, University of Stockholm, Stockholm, Sweden) of the variable selection method (Johnson, 1990).

\section{Rotary Shadowing and Electron Microscopy}

For comparative analysis in different buffers, purified polypeptides were dialyzed against phosphate-buffered saline (PBS), $0.1 \times$ PBS, or $40 \mathrm{mM} \mathrm{NH} \mathrm{HCO}_{3}$ and then centrifuged at $13,000 \times \mathrm{g}$ for $5 \mathrm{~min}$. Supernatants were supplemented with $40 \%$ glycerol. To later avoid excessive salt crystal formation, proteins in PBS were diluted with 9 volumes of $\mathrm{H}_{2} \mathrm{O}$ just before addition of glycerol. Solutions were sprayed onto freshly cleaved mica flakes and then dried under vacuum. Rotary shadowing with platinum/carbon $(95 \% / 5 \%)$ was at an angle of $7^{\circ}$, followed by shadowing with pure carbon at $90^{\circ}$ (Pesheva et al., 1989). Replicas were floated off in $\mathrm{H}_{2} \mathrm{O}$ and collected on 300-mesh copper grids. For negative staining, glycerol-free protein solutions were applied to glow-discharged carbon-coated cop- 
Table 3. Bacterial expression vector constructs

PQE.hTpr. 1-398: Encodes an N-terminal 6xHistag (MRGSHHHHHHG), spacer SPNSNSLHAA, hTpr aa1-398, and vector-derved C-terminal GTPGRPAAKLN. Total of 430 aa (including start methionine); Mr 49424. pQE. $h$ Tpr.774-1370: 6xHis-tag, SPNSNSLHAAMEOKLISEED, hTpr aa774-1370, FPGRPAAKLN. 638 aa; Mr 74319 .

DOE.hTpr.2231-2363: 6XHis-tag, IRMAARYPGSTLA, hTpr aa2231-2363. 157 aa; Mr 16867 pGEX.hTpr.1-398: N-terminal GST-tag, followed by LEVLFQGPLGSP (inker 1; PreScission recognition site underlined cleavage site between residues $Q$ and $G$ ), spacer NSL.HAA, $h T p r$ aa 1-398, AAAS. pGEX.hTpr.774-1370: GST.tag plus Inker 1, NSLHAAMEQKLISEED, hTpr aa774-1370, FPGRLERPHRD. oGEX.XiTpr.1643-7935: GST-tag, inker 1, NS, hTpr aa1637-1642, followed by aa1643-1935 of the evolutionarily highly conserved Xenopus Tpr aa sequence (Cordes et ai. 1997, 1998), and VDSSGRIVTD. pGEX.xiTpr. 1776-2176: GST-tag, linker 1, NS, xITpr aa1776-2176. RAAASS. PGEXXXTPr.2108-2363: GST-tag, inker 1, N, XITpr aa2108-2363

GST-tags of PGEX expression products were proteotytically removect calculated Mr and sequence length of resulting polypeptides: pGEX.hTpr.1-398; 414 aa; Mr 47473. pGEX.hTpr.774-1370: 630 aa; Mr 73440 . oGEX.XITpr.1643-1935: 299 aa; Mr 32209. pGEX.xITpr.1776-2176: 394 aa; Mr 42931. pGEX.xITpr.2108-2363: 253 ae. Mr 26441

per grids, allowed to adhere (15 s), washed with $\mathrm{H}_{2} \mathrm{O}(10 \mathrm{~s})$, and then stained with $2 \%$ uranyl acetate $(15 \mathrm{~s})$. Preparation of specimens for immuno-electron microscopy (EM) of cultured cells was similar to that described before (Cordes et al., 1997); after formaldehyde (FA) fixation (2.4\% in PBS, $25 \mathrm{~min})$, cells were permeabilized with Quillaja saponin (Sigma-Aldrich, Stockholm, Sweden; 0.1\% in PBS, $10 \mathrm{~min})$ or Triton $\mathrm{X}-100(0.2 \%$ in PBS, $3 \mathrm{~min})$ and then treated with blocking solution (Cordes et al., 1997; or 5\% goat serum in PBS) before incubation with antibodies. Specimens were analyzed at 60 or $80 \mathrm{kV}$ with a CM120 (Philips Electronics, Eindhoven, The Netherlands) transmission electron microscope.

\section{Gel Electrophoresis and Cross-linking}

SDS-PAGE and immunoblotting was as described previously (Cordes et al., 1997). Acrylamide gels were silver stained with Gelcode Silver Stain (Pierce) or by the method of Blum et al. (1987). For comparative analysis of cross-linking in different buffers, polypeptides were dialyzed against low salt buffer I (15 mM HEPES-NaOH, pH 7.5, $2 \mathrm{mM} \mathrm{MgCl}_{2}, 1 \mathrm{mM} \beta-\mathrm{ME}$ ), II (5 mM HEPES-NaOH, $1 \mathrm{mM}$ EDTA, $1 \mathrm{mM} \beta-\mathrm{ME}$, at $\mathrm{pH} 7.5,8.4$, and 9.0), or III (10 mM sodium phosphate, $\mathrm{pH} 7.4,1 \mathrm{mM}$ EDTA, $1 \mathrm{mM} \beta-\mathrm{ME})$, or against PBS, $0.1 \times$ PBS, or high salt buffer $(40 \mathrm{mM}$ sodium phosphate, $\mathrm{pH} 7.4,500 \mathrm{mM} \mathrm{NaCl}$ with or without $2.5 \mathrm{mM} \beta-\mathrm{ME}$ ). Protein aliquots were supplemented with 0.1 volume of $10 \times$ stock solutions of glutaraldehyde (GA; EM quality; Serva, Heidelberg, Germany), or ethyleneglycol bis[sulfo-succinimidylsuccinate] (sulfo-EGS; Pierce), prepared in buffers as for dialysis. GA stock concentrations ranged from 0.01 to $0.4 \%$ and for sulfo-EGS concentrations were $0.5,1,2,5$, and $10 \mathrm{mg} / \mathrm{ml}$. Reaction mixtures with protein concentrations of $1.0-4.5 \mu \mathrm{M}$ were incubated for 30 or 60 min at 4,11 , or $20^{\circ} \mathrm{C}$. Reactions were stopped by addition of 0.5 volume of dialysis buffer plus 1 or $2 \mathrm{M}$ glycine; 15 min later, 0.5 volume of $2 \times$ SDS-protein sample buffer was added.

\section{Yeast Two-Hybrid Assays}

S. cerevisiae strains Y190 (Mat a) and Y187 (Mat $\alpha$; both from Clontech) were used for single transformations with pAS-1, and pACT-2 vectors, respectively. Cells were mated and diploid cells analyzed for lacZ activation. Occasionally, protein interactions were studied in haploid cells (Y190), double-transformed with pairs of vectors. 5-Bromo-4-chloro-3-indolyl-D-galactoside (X-Gal) filter lift assays were performed according to the Yeast Protocols Handbook PT3024-1 (Clontech). For rating of levels of X-Gal hydrolysis, up to 15 pairs of binding domain (BD) and activation domain (AD) constructs were analyzed in parallel, including several pairs routinely used as calibration standards.

\section{Sucrose Density-Gradient Centrifugation and Gel Filtration Chromatography}

For sedimentation velocity experiments, linear gradients of $5-30 \%$ sucrose were made in PBS plus "Complete" protease inhibitor cocktail (Roche). Cells were permeabilized with $0.3 \%$ Triton X-100 in PBS plus inhibitors and centrifuged at $13,000 \times g$ for $15 \mathrm{~min}$ at $4^{\circ} \mathrm{C}$. 500 $\mu \mathrm{l}$ of supernatant was loaded per gradient (total volume $10.5 \mathrm{ml}$ ). Bovine serum albumin (BSA; 4.4 Svedberg units [S]), catalase (11.3 $\mathrm{S})$, and thyroglobulin (16.5 S) were used in parallel gradients as standards with known sedimentation coefficients (Handbook of Biochemistry, 1970). Centrifugation at $4^{\circ} \mathrm{C}$ and precipitation of 0.5-ml fractions was as described previously (Cordes et al., 1993). Gel filtration chromatography was performed at $4^{\circ} \mathrm{C}$ on a Superose 6 column (Pharmacia) equilibrated with PBS plus protease inhibitor cocktail and loaded with $\sim 0.4 \mathrm{ml}$ of $13,000 \times g$ cell extract supernatant. Fractions of $0.25 \mathrm{ml}$ were collected at $0.25 \mathrm{ml} / \mathrm{min}$ and precipitated overnight with 4 volumes of methanol at $-20^{\circ} \mathrm{C}$. Standard proteins used for calibration were human immunoglobulin (Ig) M (Stokes radius, $R_{S}$, of $125 \AA$ ), bovine thyroglobulin (85 $)$ ), horse ferritin (61 $\AA$ ), bovine catalase (52.2 $\AA$ ), and rabbit aldolase (48.1 Å; Takashima et al., 1988; Handbook of Gel Filtration: Principles and Methods, 1998). The position of the void volume was determined by with the use of Blue Dextran 2000. Human IgM was from Sigma; other standards were from Pharmacia. The equation for the calibration curve was determined by least square fitting with the use of the Matlab 5.2 software (www.mathworks.com/products/ matlab). The molecular weight $(\mathrm{M})$ of soluble Tpr was determined by the equation: $\mathrm{M}=s N 6 p \eta R_{S}(1-v \rho)^{-1}$ (Siegel and Monty, 1966; Cantor and Schimmel, 1980), where $s$ is the sedimentation coefficient (expressed in $\mathrm{S}$ units; $1 \mathrm{~S}=10^{-13} \mathrm{~s}$ ), $N$ is Avogadro's number, $\eta$ the viscosity, and $\rho$ is the density of water at $20^{\circ} \mathrm{C}$. The partial specific volume of Tpr, $v$, was assumed to be similar to that of other proteins such as myosin $\left(\sim 0.73 \mathrm{~cm}^{3} \mathrm{~g}^{-1}\right.$; Cantor and Schimmel, 1980).

\section{Transfection, Immunofluorescence Microscopy, and Assessment of NPC Binding}

Human cells of lines PLC (ATCC CRL 8024) and 293 (ATCC CRL 1573) were cultured as described before (Cordes et al., 1998). Cells were split $1 \mathrm{~d}$ before transfection and were transfected at a density of $30-50 \%$ with the use of Superfect (Qiagen) or FuGene6 Transfection Reagent (Roche). Cells were analyzed $18-24 \mathrm{~h}$ posttransfection. Standard fixation of cells in $2.4 \%$ FA in PBS was for $20 \mathrm{~min}$ and subsequent permeabilization was with $0.3 \%$ Triton X-100 in PBS for $3 \mathrm{~min}$. For permeabilization before fixation, cells were submerged in PBS (plus or minus $5 \mathrm{mM} \mathrm{MgCl}$ ) with $0.3 \%$ Triton X-100 or $0.3 \%$ Quillaja saponin for $3 \mathrm{~min}$, washed in PBS $(<5 \mathrm{~s})$, and fixed with $3.7 \%$ FA in PBS (15 min); subsequent washings in PBS with and without $\mathrm{NH}_{4} \mathrm{Cl}$ followed standard procedures but omitted further detergent treatment. Additional salt extraction before fixation was in PBS plus $0.36 \mathrm{M} \mathrm{NaCl}$ (5 min). Blocking (1\% BSA in PBS, $10 \mathrm{~min}$ ), subsequent incubation with antibodies, and mounting of nondehydrated specimen, was as described by Cordes et al. (1998). For confocal microscopy, an LSM 510 (Zeiss, Oberkochen, Germany) was used. After joint transfection and growth on pairs of coverslips, cell transfection efficiency versus cell percentage with residual $\alpha$-Myc stain at NPCs was determined by in-parallel evaluation of postfixation- and prefixation-permeabilized specimen. For rating of relative $\alpha$-Myc-staining intensity at NPCs, cells were transfected with up to 16 different constructs in parallel, including up to five constructs routinely used as calibration standards, representing i) NPC binding, ii) binding-impaired, and iii) non-NPC-binding Tpr polypeptides. Specimens were randomly encoded, evaluated by IF microscopy for ranking of nuclear rim staining intensity, and decoded again. With the use of different cell passages, rating was performed at least twice for each construct. 
Figure 1. Purification of recombinant Tpr polypeptides and CD analysis. (A) Prediction of coiledcoil-forming regions of human Tpr with the use of the Paircoil program (Berger et al., 1995). The vertical scale represents relative coiled-coil probability; the horizontal scale represents Tpr's aa sequence. (B) Schematic representation of Tpr and fragments thereof. The white rectangle with gray boxes represents the N-terminal domain of 1630 aa; the hatched rectangle represents the C-terminal domain of 733 aa Gray boxes represent 14 clusters of consecutive copies of the HR motif for which $>90 \%$ coiled-coil probability is predicted. The two C-terminal segments represent polypeptides of Xenopus Tpr with aa numbering in accordance to the evolutionary conserved human sequence. (C) Purified polypeptides separated by SDSPAGE and stained by Coomassie blue. Relative masses of marker proteins (left lane) are 205, 116 , 97, 84, 66, 55, 45, and $36 \mathrm{kDa}$. Polypeptides 1-398 and 774-1370 shown here include a His tag. GST tags initially fused to 1643 1935 and 2108-2363 have been proteolytically removed. (D) CD spectra of polypeptides recorded at $20^{\circ} \mathrm{C}$ in $40 \mathrm{mM}$ sodium phosphate buffer at $\mathrm{pH}$ 7.4. Vertical scales represent ellipticity expressed in millidegrees; horizontal scales represent wavelength range of measurements. Pathlength of cuvettes was $1 \mathrm{~mm}$ for 1-398 (protein concentration $\sim 1.9 \mu \mathrm{M})$ and 774-1370 $(\sim 1.6 \mu \mathrm{M})$, and $0.05 \mathrm{~mm}$ for 1643-1935 $(\sim 4.5 \mu \mathrm{M})$ and $2108-2363(\sim 6.0$ $\mu \mathrm{M})$. No conspicuous differences of spectra for respective Tpr segments were noted at different temperatures or between non- or His-tagged polypeptide versions.

\section{RESULTS}

\section{Amino-Terminal Domain Segments of Tpr Are Dominated by $\alpha$-Helices, Are of Rod-like Shape, and Homodimerize In Vitro}

To gain insight into the basic secondary and ultrastructural characteristics of Tpr, polypeptides representing different segments of Tpr's N- and C-terminal domain were synthesized in bacteria and then analyzed by CD spectroscopy, chemical cross-linking, and EM. Figure 1 presents a collection of Tpr polypeptides purified to near homogenity under nondenaturing conditions. Attempts to purify full-length Tpr from various mammalian tissues, or to isolate the fulllength $\mathrm{N}$ - and C-terminal domains from bacterial or insect expression systems, did not yield sufficiently high amounts of undegraded protein (Brettel, Hase, Herrmann, Krohne, and Cordes, unpublished data).

CD spectra recorded for both N-terminal domain segments were almost indistinguishable but clearly differed from the C-terminal polypeptides, which also had common characteristic features (Figure 1D); CD spectra of other Tpr polypeptides (Tpr 1-135, 1776-2176, 2231-2363; Cordes, un- published results) were equally characteristic of either the $\mathrm{N}$ - or C-terminal domain. Those for the C-terminal domain segments were found to be made up mainly of spectral components corresponding to a mixture of $\beta$-turns and random coils $(\sim 50 \%$ of the entire structure) and parallel $\beta$-sheets $(\sim 30 \%)$. By contrast, the $\mathrm{N}$-terminal polypeptides were found to be dominated by $\alpha$-helices, resulting in positive and negative ellipticity peaks at 195, 208, and $222 \mathrm{~nm}$. The spectra for Tpr 1-398 and 774-1370 (pQE versions, see Table 3) were calculated to represent $\alpha$-helical contents of $\sim 70$ and $\sim 79 \%$, respectively, which are similar to values of $\alpha$-helical content (79 and $85 \%$ ) determined by a secondary structure prediction algorithm (Frishman and Argos, 1997).

Electron micrographs obtained after heavy metal rotary shadowing of the recombinant polypeptides revealed an extended rod-like shape of the N-terminal domain segments. Tpr 1-398 appeared as mostly rectilinear, only seldomly kinked rods with a thickness of 2-3 nm and a rather uniform length of $51 \pm 3 \mathrm{~nm}$ (Figure 2A, pQE version of Tpr 1-398); similar results were obtained for both nontagged and His-tagged versions of the polypeptide. Images of Tpr polypeptide 774-1370 also revealed clearly rod-shaped mol- 

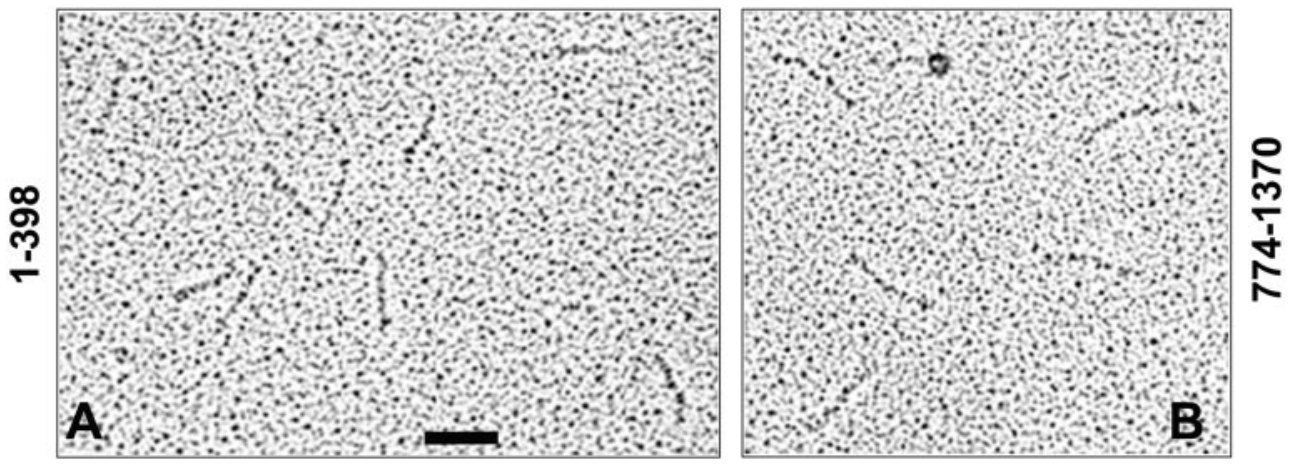

Figure 2. Rotary shadowing electron microscopy reveals a rod-shaped ultrastructure of $\mathrm{N}$-terminal domain segments. Recombinant Tpr 1-398 (A) and 7741370 (B) were rotary shadowed with platinum at low angle $\left(7^{\circ}\right)$. Note the thin, rod-like structures of rather uniform length $(\varnothing$ of $\sim 51 \mathrm{~nm})$ in A, and of similar appearance, but varying length (here $61-77 \mathrm{~nm}$ ) in B. Bar, $50 \mathrm{~nm}$ for $\mathrm{A}$ and $\mathrm{B}$.

ecules (Figure 2B), which, however, varied significantly in length, commonly ranging from $\sim 40$ to $\sim 65 \mathrm{~nm}$ and only rarely reaching $70-80 \mathrm{~nm}$. Although usually rectilinear, Tpr 774-1370 rods sometimes also appeared to be curvilinear, kinked, or folded, and occasionally revealed bifurcations at one of their ends (Cordes, unpublished results). At higher
A

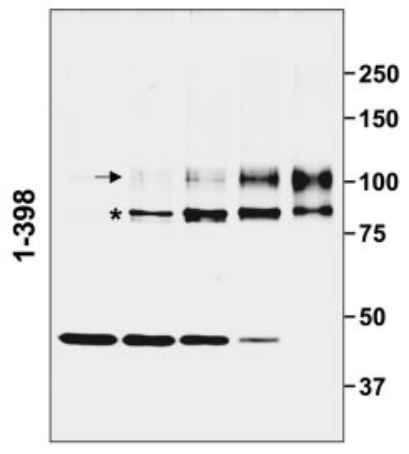

C

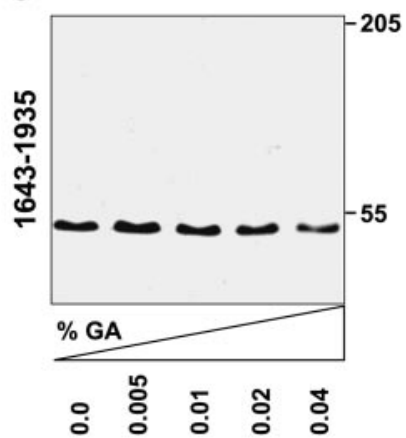

B

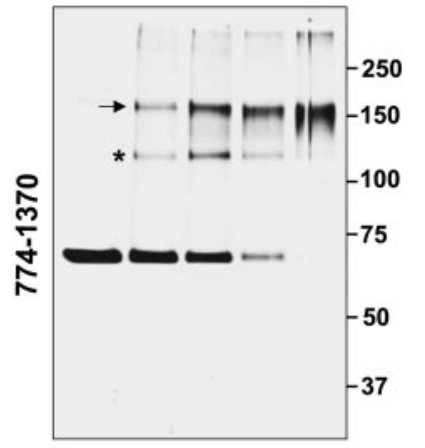

D

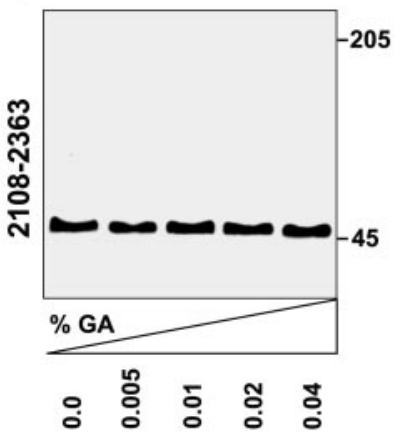

Figure 3. Chemical cross-linking studies show homodimeric protein-protein interactions in vitro between recombinant polypeptides of Tpr's N-terminal but not C-terminal domain. Polypeptides in low salt buffer II at pH 8.4 (A), low salt buffer I at pH 7.6 (B), or in PBS, $\mathrm{pH} 7.4(\mathrm{C}$ and $\mathrm{D})$ were incubated in the absence or presence of increasing amounts of GA $(0.0-0.04 \%)$, separated by SDS-PAGE, and silver stained. Molecular mass standards in kilodaltons are indicated at the right. Arrows and asterisks (A and B) mark modification or folding variants of cross-link products at the approximate dimer position. molar concentrations, Tpr 1-398 as well as Tpr 774-1370 appeared randomly entangled without apparent higher order regularity. Occasionally, the His-tagged versions of both polypeptides aggregated into "starfish"-like clusters, where several rods appeared radially arranged and connected with one of their ends to a common center (Cordes, unpublished results); however, because these assemblies were never observed with the nontagged polypeptide versions, they were considered an artifact of the His tag. By contrast, the C-terminal domain Tpr polypeptides showed no regular structures to be unambiguously identified in metal-shadowed specimens.

The rod-shaped appearance suggested that the N-terminal domain segments form homomeric coiled coils. To examine this inference, the polypeptides were incubated in various buffers (see MATERIALS AND METHODS) in the presence of cross-linkers and then analyzed by SDS-PAGE. Increasing concentrations of GA in low salt buffers diminished the intensity of the monomeric polypeptide bands at $46 \mathrm{kDa}$ (Tpr 1-398; Figure 3A), and 69 kDa (Tpr 774-1370; Figure 3B) and gave rise to bands with apparent molecular masses of 84 and $101 \mathrm{kDa}$ (Tpr 1-398) and of 120 and $153 \mathrm{kDa}$ (Tpr 774-1370). Independent of variations in SDS-PAGE conditions and use of different size references, size relations between a polypeptide at the monomer position and its two cross-link products remained constant: ratios between relative sizes of Tpr 1-398 bands were determined to be 1:1.8 $( \pm 0.05): 2.1( \pm 0.15)$, and 1:1.75 $( \pm 0.05): 2.15( \pm 0.1)$ for Tpr 774-1370. This suggested that the higher molecular mass bands both represent the dimeric protein but as cross-linkermodified folding variants. The same patterns were obtained when incubations were performed as time courses with a fixed concentration of GA or when the cross-linker SulfoEGS was used (Hase and Cordes, unpublished results).

In low salt buffers at pH 7.6 (as in Figure 3B), additional cross-link products of higher molecular mass were observed in only minor amounts and did not occur at $\mathrm{pH} 8.4$ (Figure 3A) or 9.0, indicative that under the latter conditions both Tpr 1-398 and Tpr 774-1370 behaved exclusively as dimers. In contrast, GA treatment of Tpr 1-398, Tpr 774-1370, and smaller segments such as Tpr 1-135 in buffers of moderate to high salt concentrations at pH 7.4-7.6, resulted in numerous cross-link intermediates and finally in a degree of cross-linking that precluded resolution by SDS-PAGE. However, polypeptides in neither of these nor other buffers revealed any filamentous structures of higher order regularity in the EM when studied as metal-shadowed (see above) or negatively stained specimens (not shown). Therefore, it was considered most likely that in 
Figure 4. Yeast two-hybrid analysis reveals in-parallel and in-register homodimerization between Tpr's N-terminal regions in vivo. Schemes of Tpr, and of segments encoded by expression vectors, are in adaptation to Figure $1 \mathrm{~B}$. (A) Interactions between identical Tpr segments fused to yeast Gal4-BD and Gal4-AD, respectively. Asterisk, because fusion product BD 2069-2363 causes autoactivation of reporter genes, the result for the non-autoactivating pair of $\mathrm{AD}$ 2069-2363 and BD 1622-2363 is given instead. Marks indicate lacZ reporter gene activation; light blue $(+)$, blue $(++)$, and dark blue $(+++)$ yeast colonies reflect different amounts of hydrolyzed X-Gal; cells without reporter gene activation $(-)$ remain white. Each interaction experiment included analysis of four clones and was repeated at least twice. Superscript 1, level of XGal hydrolysis varied between different experiments or individual clones within ranges indicated. (B) Selection of experiments in which BD- and ADfusion products were tested reciprocally for interaction with other segments. Note that nonoverlapping segments generally do not interact; low levels of XGal hydrolysis were noted only when AD 774-1370 was coexpressed with either BD 1-398 or BD 305-775 (superscript 2) but not in reciprocal combinations, i.e., AD 1-398 plus BD 774-1370, or AD 305-775 plus BD 774-1370.

(C) Further dissection of the Nterminal domain reveals specificity of interaction between isogenic, HR-containing clusters. $\mathrm{BD}$ - and AD-fusions with two or

three HR clusters were tested reciprocally for interaction with all other constructs. Asterisk, BD 397-663 causes autoactivation (a) of reporter genes (also observed for BD 401-651 and 371-663; Hase and Cordes, unpublished results); in contrast, autoactivation does not occur with BD fusions of larger segments containing HR 5-6 or with AD fusions of HR 5-6; interaction between the latter and BD 305-775 results in high level $(+++)$ X-Gal hydrolysis.

vitro cross-linking of Tpr in the presence of elevated salt concentrations also included random intermolecular bond formation between activated $\alpha$ - and $\gamma$-amino groups. In contrast, the electrophoretic mobility of Tpr's C-terminal domain segments (Figure 3, C and D) remained unaffected even at high GA concentrations and in different buffers.

\section{Homodimerization of Tpr Occurs in Parallel and in Register}

To study homodimerization of Tpr in more detail, we used the yeast two-hybrid system (Fields and Song, 1989) which proteins of interest. The proteins are coexpressed in a yeast host cell as fusion proteins, with one protein fused to the AD interaction between the proteins reconstitutes the activator, leading to transcription of reporter genes. This approach has been used previously to study dimerization of other coiledcoil proteins (Ye and Worman, 1995; Carpenter and Ip, 1996; Leung and Liem, 1996; Meng et al., 1996).

With the use of lac $Z$ as the reporter gene and monitoring $\beta$-galactosidase activity in a colorimetric filter assay, we could demonstrate that identical segments of Tpr's N-termi- permits examination of in vivo interactions between two and the other to the DNA BD of a transcription activator; 
nal domain were capable of homodimerizing in vivo (Figure 4A). In contrast, N-terminal polypeptides that did not share overlapping regions generally did not interact with each other (Figure 4B). These results indicated that interactions between Tpr molecules result in parallel rather than antiparallel homodimers. Furthermore, no interactions were observed between polypeptides of the C-terminal domain (Figure $4 \mathrm{~A}$ ) or between Tpr fragments and either coiled-coil protein vimentin or an HR-containing segment of nuclear lamin C used as control.

Further truncation of Tpr's N-terminal domain into smaller units containing only two or three HR clusters revealed that dimerization between Tpr polypeptides occurs in register rather than in a staggered arrangement. The small HR-containing segments bound only to segments of the same kind or to such containing at least one common HR cluster. In contrast, binding to HR clusters of nonoverlapping regions was not observed (Figure 4C).

Interactions between full-length Tpr or between polypeptides representing the full-length $\mathrm{N}$-terminal domain (fusion proteins of $>200 \mathrm{kDa}$ ) could not be studied by the yeast two-hybrid approach; to a certain extent this appeared to be due to aggregation and impaired nuclear import of large hTpr molecules in the yeast cell (Hase and Cordes, unpublished results). Additionally, sterical constraints interfering with transcription activation were likely (Newman et al., 2000).

\section{A Surplus of Tpr in Transfected Cells Does Not Self- Assemble into Stable Homopolymeric Filaments but Forms a Soluble Pool}

To investigate whether Tpr or any of its segments can form stable homopolymeric filaments in vivo, we overexpressed the full-length protein and various deletion mutants in transiently transfected human cells of line PLC and analyzed these by confocal IF microscopy and cell fractionation. The recombinant polypeptides were recognized via their myc tag, with the use of mAb 9E10. The possibility that the N-terminal myc tag may have a disturbing effect on Tpr's assembly properties was minimized by control experiments yielding similar results with nontagged, full-length recombinant hTpr that had been expressed in monkey and rodent cell lines. In these cells, hTpr had been visualized with $\mathrm{mAb}$ 203-37 which recognizes the human protein but is not equally reactive with Tpr homologues of other mammals (Cordes, unpublished results).

In PLC cells fixed with FA and then permeabilized by detergent, wild-type Tpr was found primarily at the nuclear periphery, with no or only traces of additional labeling seen deep within the nuclear interior; indistinguishable IF results were obtained with different antibodies specific for epitopes within either the $\mathrm{N}$ - or C-terminal domain of hTpr (Figure 5). Similarly, also full-length recombinant Tpr was generally found associated with the nuclear rim, previously shown to correlate with binding to or near individual NPCs (Bangs et al., 1998; Cordes et al., 1998). In addition, however, in transfected cells with high amounts of the recombinant protein, the surplus of Tpr was distributed throughout the extranucleolar nuclear interior (Figure 5, top). To assess to which degree this latter pool of Tpr may be associated with intranuclear structures, cells on coverslips were treated with
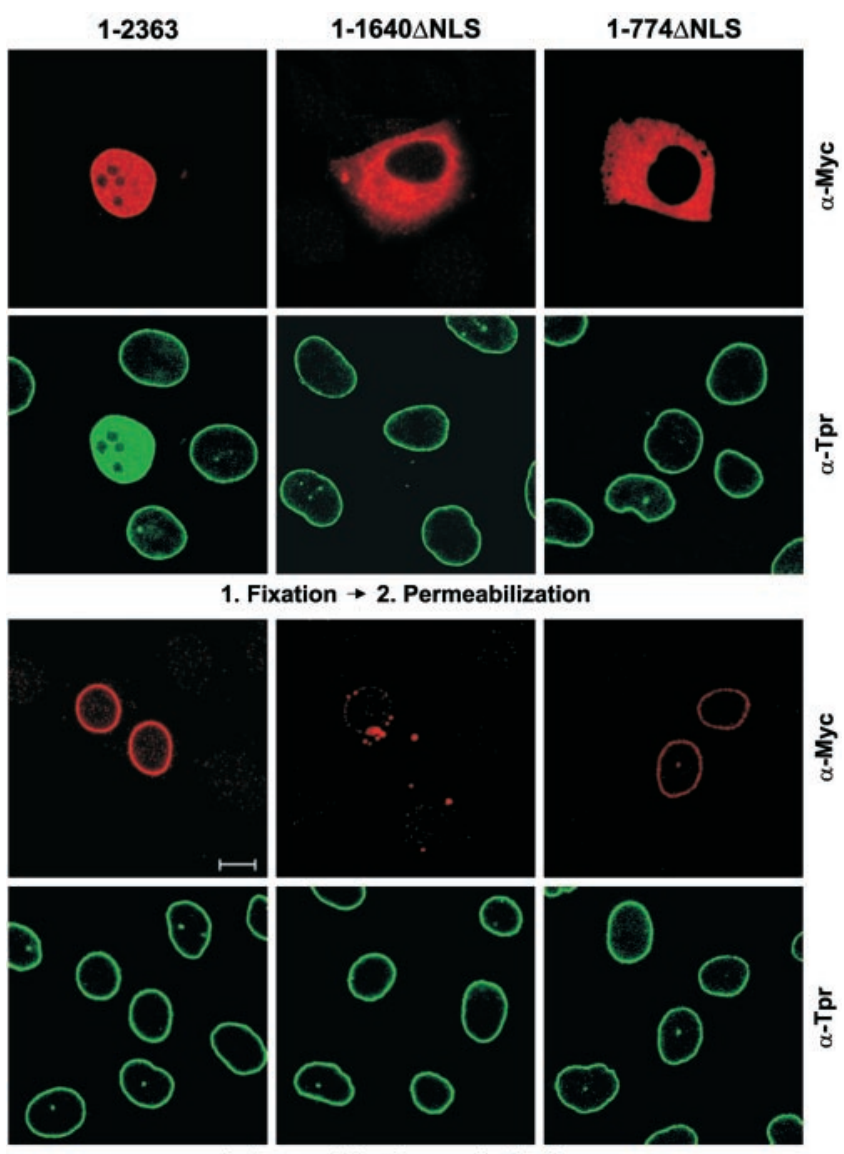

1. Permeabilization $\rightarrow$ 2. Fixation

Figure 5. Surplus recombinant Tpr not bound to NPCs adds to a soluble pool of wild-type Tpr. Confocal IF micrographs of human PLC cells transiently transfected with expression vectors encoding full-length Tpr (1-2363), and deletion mutants making

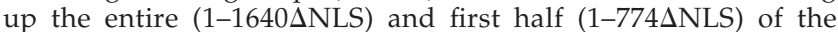
NLS-free N-terminal domain. Cells on pairs of coverslips were permeabilized with Triton X-100 after and before FA fixation. Double labeling was with mAb 9E10 against the N-terminal myc tag of recombinant polypeptides $(\alpha$-Myc) and rabbit antibodies against aa 2063-2084 of hTpr ( $\alpha$-Tpr). Immunostaining of wildtype Tpr near-exclusively at the nuclear periphery was also observed with guinea pig antibodies against hTpr aa 1622-1640 and with $\mathrm{mAb}$ 203-37 against Tpr's N-terminal domain. Plane of focus was at or near the cells' nuclear equator. Note that in addition to nuclear rim staining, high synthesis rates of Tpr 1-2363 result in accumulation within the nuclear interior; this intranuclear pool is quantitatively released by brief permeabilization of cells before fixation. Similarly, Tpr rod polypeptides accumulated within the cytoplasm are largely extracted by detergent treatment, with the exception of aggregate-like cytoplasmic structures in cells expressing 1-1640 $\Delta$ NLS (bottom $\alpha$-Myc micrograph, residual aggregates from two transfected cells). Bar, $10 \mu \mathrm{m}$ for all micrographs.

different extraction buffers before fixation and confocal IF. Unexpectedly, the extranucleolar pool of Tpr could be quantitatively released by brief permeabilization of cells with the nonionic detergent Triton X-100 in PBS (Figure 5, bottom). Identical results were obtained for cells permeabilized in the 
Figure 6. Gel filtration chromatography and sucrose density-gradient centrifugation of soluble Tpr. (A) Immunoblot detection of wild-type Tpr in soluble fractions of mammalian cells. Total proteins from nontransfected PLC and 293 cells were separated into $13,000 \times g$ supernatants released by permeabilization with Triton X-100 (S) and residual, nonextracted proteins $(\mathrm{P})$; $\mathrm{S}$ lanes were loaded at $10 \times$ higher ratio of fraction than $\mathrm{P}$ lanes. Tpr and two putative degradation products (arrowheads; see also Cordes et al., 1997) were detected by mAb 203-37 ( $\alpha$-Tpr-N), which binds to an epitope located between aa 1370 and 1623 of hTpr. Identical results were obtained with antibodies against aa 1622-1640 of hTpr, representing the Cterminal end of the rod domain. (B) 13,000 $\times g$ supernatants from nontransfected PLC were fractionated by gel filtration chromatography and analyzed by immunoblotting with the use of rabbit antibodies against aa 2063-2084 of hTpr ( $\alpha$-Tpr-C) and $\mathrm{mAb}$ 203-37. Peak positions for void volume (v) and reference proteins of known Stokes radii (IgM, $125 \AA$; thyroglobulin, $83 \AA$; ferritin, $61 \AA$; catalase, $52.2 \AA$, aldolase, $48.1 \AA$ ) are indicated by arrows. Peaks of full-length soluble Tpr were repeatedly found in fractions 7 and/or 8, corresponding to an average Stokes radius of $161 \pm 6 \AA$. Representative gels shown here were loaded with fractions of odd numbers only. (C) 13,000 $\times g$ supernatants from nontransfected PLC and 293 cells (top fluorographs) and from 293 cells expressing myc-tagged Tpr 1-2363 (bottom fluorograph) were fractionated by sucrose gradient centrifugation and analyzed by immunoblotting with the use of mAb 203-37 or mAb 9E10 ( $\alpha$-Myc). Only upper portions of filters are shown. Peak positions for reference proteins of known sedimentation coefficient (BSA, $4.4 \mathrm{~S}$; catalase, $11.3 \mathrm{~S}$; thyroglobulin, 16.5 S) are indicated by arrows. Note that both wild-type and recombinant Tpr are in peak fractions corresponding to mean $\mathrm{S}$ values of $7.5 \mathrm{~S}$ for the intact protein and smaller $\mathrm{S}$ values $(\sim 6.5 \mathrm{~S})$ for products of Tpr degradation during gradient centrifugation.

absence or presence of up to $5 \mathrm{mM} \mathrm{MgCl}_{2}$, the latter reported to stabilize NPC-associated intranuclear filaments in Xenopus oocytes (Arlucea et al., 1998). In contrast, staining for recombinant Tpr at the nuclear rim remained unaffected even when detergent extraction was followed by incubations in buffers of $0.5 \mathrm{M}$ salt before fixation.

Recombinant polypeptides, which make up the full-length

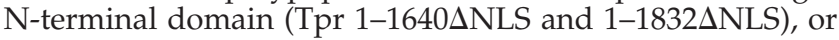
its first half (Tpr 1-774 $\Delta$ NLS), lack an NLS (Cordes et al., 1998) and accumulate within the cytoplasm. These cytoplasmic pools of Tpr polypeptides were also largely extracted by treatment with Triton X-100; with the exception of residual aggregate-like structures often present in the cytoplasm of cells expressing the full-length N-terminal domain (Figure 5). With the use of Quillaja saponin, a triterpenoid glycoside with comparably mild nonionic detergent properties, extraction of the cytoplasmic pool was equally quantitative. In a smaller population of transfected cells expressing $1-774 \Delta$ NLS, weak to moderate residual nuclear rim staining was observed. However, detergent-resistant structures of marked filamentous appearance were not seen with this or any other Tpr derivative used in this study.

After permeabilization of nontransfected human cells of lines PLC and 293 with Triton X-100, we had identified a minor soluble pool of wild-type Tpr. This pool represented $<5 \%$ of total Tpr in an interphase cell population (Figure 6A). Characterization by gel filtration chromatography and sucrose gradient centrifugation had revealed that soluble full-length Tpr was in a near-monodisperse form, with a Stokes radius of $\sim 161 \AA$ ( $\pm 6 \AA$; Figure $6 \mathrm{~B})$ and a peak sedimentation coefficient of $\sim 7.5 \mathrm{~S}$ (Figure $6 \mathrm{C}$, top fluorographs). These results allowed us to calculate (for details, see MATERIALS AND METHODS) a corresponding molecular weight of $\sim 509,000( \pm 19,000)$ for the soluble full-length form. This value is very close to the sequence-deduced value of 534.670 predicted for a homodimer of full-length Tpr. Truncated forms of soluble Tpr, which may represent degradation products, were noted to have peak distributions corresponding to similar Stokes radii but lower sedimentation coefficients when compared with those of the full-length protein. These shorter forms of Tpr apparently contain the full-length N-terminal but lack parts of the C-terminal domain (Figure 6B; see also Cordes et al., 1997). It remains to be investigated whether monomeric full-length and truncated Tpr polypeptides may coexist in dimers and consequently influence peak distributions in gel filtration chromatography and velocity sedimentation. 

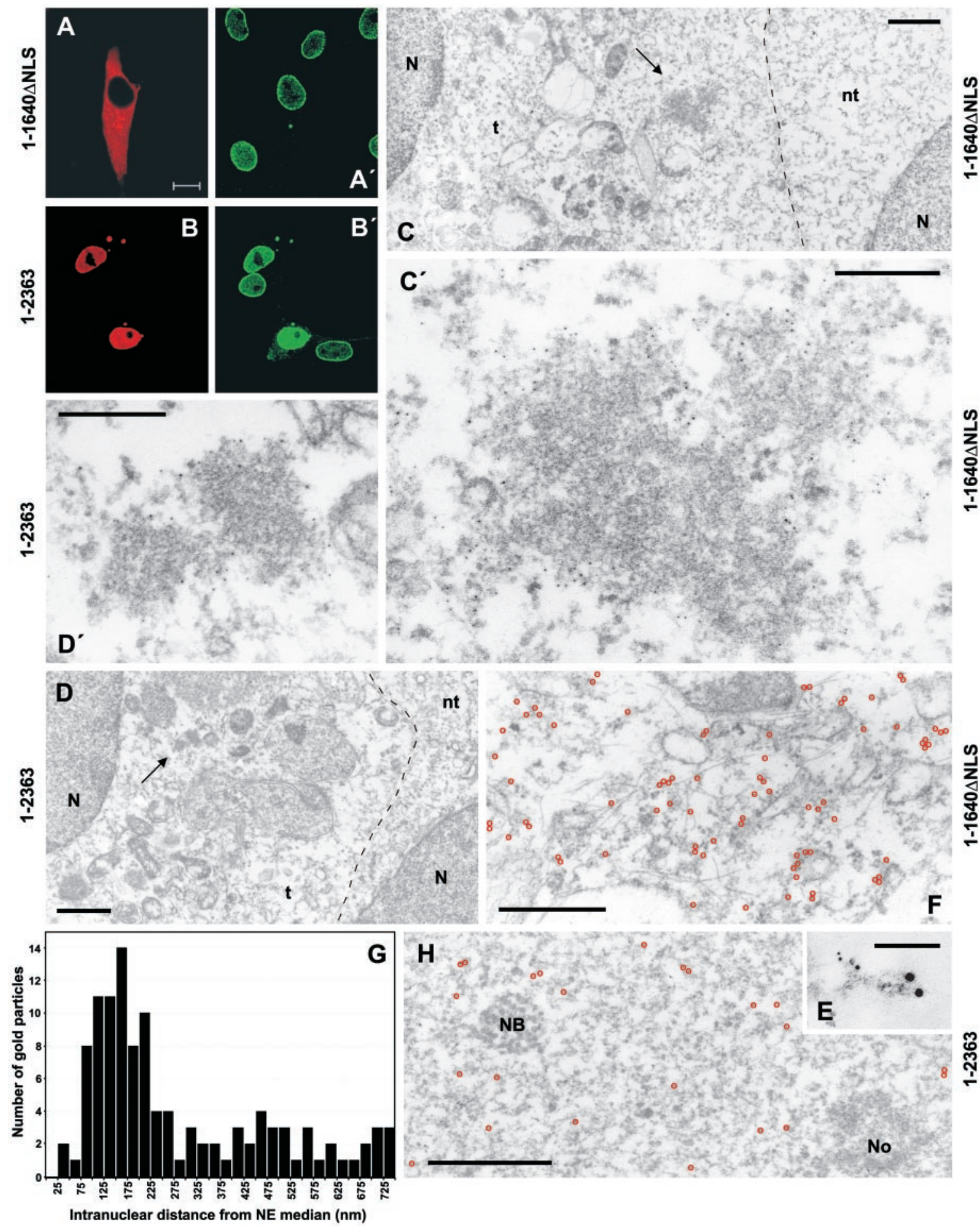

Figure 7. Immuno-EM analysis of cytoplasmic and nuclear accumulations of recombinant Tpr polypeptides in transfected human cells of line 293. (A-B') As reference, confocal IF micrographs of 293 cells, transiently transfected with vectors encoding full-length Tpr (1-2363) and deletion mutant 1-1640 $\mathrm{NLS}$, are shown. Cells, FA-fixed before permeabilization, were double labeled with mAb 9E10 against N-terminal myc tags (A and B) and rabbit antibodies against aa 2063-2084 of hTpr ( $\mathrm{A}^{\prime}$ and B'). Because 293 nuclei often are rather flat, and in general significantly smaller than those of PLC cells from cultures of similar density, the optical sections shown do not all correspond to a near-equatorial nuclear plane. (C-D') Immuno-EM of cytoplasmic aggregates of recombinant Tpr 1-1640 with mAb 9E10 and 5-nm gold-coupled secondary antibodies. Gold-decorated aggregates demarked by arrows in overviews (C and D) of 
To determine whether detergent-extractable recombinant Tpr 1-2363 represented a similarly monodisperse soluble form, extracts from transfected 293 cells were also analyzed by sucrose gradient centrifugation (293 cells were chosen because they could be transfected more efficiently than PLC cells). Indeed, soluble recombinant Tpr was found to have a similar peak sedimentation value of $\sim 7.5 \mathrm{~S}$ (Figure 6C, bottom fluorograph).

\section{Immunogold Labeling of Recombinant Tpr in Transfected Cells}

In 293 cells with high amounts of recombinant Tpr, the protein is not only i) bound to NPCs and ii) present in a soluble intranuclear pool but iii) often is also found in stable, aggregate-like cytoplasmic structures. As in PLC cells, similar cytoplasmic structures were also seen upon overexpression of the full-length N-terminal domain (Figure 7A-B' ${ }^{\prime}$ ). To determine whether these structures exhibit any regular morphology, we studied transfected 293 cells by pre-embedding immuno-EM. Cells were fixed before permeabilization both for better structure preservation and to allow concomitant visualization of all pools of recombinant Tpr.

With the use of myc tag-specific mAb 9E10 and 5-nm gold-coupled secondary antibodies, transfected cells were detected by specific and dense gold labeling of their respective pools of recombinant Tpr. In contrast, no or only sporadic gold grains per section plane were found in neighboring nontransfected cells. Gold-decorated cytoplasmic aggregations of Tpr 1-1640 $\Delta$ NLS (Figure 7, C and $C^{\prime}$ ) and Tpr 1-2363 (Figure 7, D and D') were found in several cells. Aggregate clusters studied in detail $(n=7$ for Tpr 1-1640 $\Delta$ NLS, $\mathrm{n}=5$ for Tpr 1-2363; for clusters of diameter $\geq 0.5 \mu \mathrm{m}$ ) revealed an electron-dense web of entangled molecules. Despite analysis of serial sections through some aggregates, neither rectilinear filaments nor paracrystalline assemblies were observed. Detergent-resistant intranuclear dot- or plaque-like structures, seldomly observed by IF microscopy in a minor population of 293 cells with recombinant Tpr 1-2363 (not shown), were not found in the EM specimen.

Figure 7 (facing page). transfected cells (t) are shown at higher magnification in $\mathrm{C}^{\prime}$ and $\mathrm{D}^{\prime}$, revealing an electron-dense web of entangled molecules but no rectilinear filamentous assemblies. Hatched lines demark cell boundaries between transfected and nontransfected (nt) cells. N, nucleus. (E) Double label immuno-EM of recombinant 1-2363 polypeptides with mAb 9E10 (5-nm gold) and rabbit antibodies against Tpr aa 2063-2084 (10-nm gold). (F) Immuno-EM of $1-1640 \Delta$ NLS after reaction with mAb 9E10 revealing random distribution in cytoplasmic areas free of Tpr aggregates and no preferential accumulation at endogenous cytoskeletal elements. Positions of 5-nm gold grains are highlighted by red circles. ( $\mathrm{G}$ and $\mathrm{H}$ ) Immuno-EM of Tpr 1-2363 after reaction with mAb 9E10 revealing intranuclear accumulation near the nuclear periphery $(G)$ but random distribution deep within the nuclear interior $(\mathrm{H})$. Nuclear envelope-proximal positions of gold grains $(n=114)$ in randomly chosen nuclear sections of transfected cells were determined with respect to a perpendicular from the median between inner and outer nuclear membrane (G). 5-nm gold grains in $\mathrm{H}$ highlighted by red circles. NB, nonspecified nuclear body; No, nucleolus. Bars, 10 $\mu \mathrm{m}\left(\mathrm{A}-\mathrm{B}^{\prime}\right), 1 \mu \mathrm{m}(\mathrm{C}$ and $\mathrm{D}), 500 \mathrm{~nm}(\mathrm{~F}$ and $\mathrm{H}), 250 \mathrm{~nm}\left(\mathrm{C}^{\prime}\right.$ and $\left.\mathrm{D}^{\prime}\right)$, $100 \mathrm{~nm}(\mathrm{E})$.
On sections of cells with Tpr 1-1640 2 NLS, numerous gold grains in addition to those decorating the aggregates were found randomly distributed throughout the cytoplasmic compartment, with no apparent accumulation at endogenous cytoskeletal (Figure 7F) or other structures. Similarly, in cells containing recombinant Tpr 1-2363, additional gold grains sporadically found in the cytoplasm did not appear structure associated (an example of a double-labeled, cytoplasmic Tpr 1-2363 molecule is shown in Figure 7E). By contrast, recombinant Tpr in the nucleus clearly accumulated at the nuclear periphery near NPCs (Figure 7G). With respect to a $250-\mathrm{nm}$ perpendicular from an imaginary median between inner and outer nuclear membrane, most gold particles were found at a mean distance of $\sim 155 \mathrm{~nm}$ (defined as NPC-proximal pool). This is equivalent to $\sim 135 \mathrm{~nm}$ from the inner NPC annulus and consequently more distal to the NPC than the terminal ring of the nuclear basket. However, considering Tpr's length and that immunolabeling of the recombinant protein occurred via its $\mathrm{N}$-terminal myc tag, the peak distribution of gold grains does not exclude a direct interaction between Tpr and terminal ring.

Gold particles deeper within the nuclear interior (intranuclear pool) appeared randomly distributed without apparent structure association (Figure $7 \mathrm{H}$ ). The transition from the steep and nearsymmetric distribution curve for the NPC proximal pool, to the flat distribution gradient of the intranuclear pool, was characterized by high curvature, indicative of a rather sharp transition between both pools (Figure 7G).

In contrast to the dramatic ultrastructural alterations of the nucleus after overexpression of coiled-coil protein NuMA (Gueth-Hallonet et al., 1998), similar effects were not observed upon nuclear accumulation of Tpr.

\section{A Single HR Cluster-containing Segment Is Essential for NPC Association of Tpr}

Recent studies had shown that different segments of the first but neither the second half of Tpr's N-terminal domain nor the C-terminal domain are capable of binding to or near NPCs (Bangs et al., 1998; Cordes et al., 1998). However, because each of the $\mathrm{N}$-terminal segments contained putative coiled-coil as well as noncoiled regions, it remained unknown whether HRs or other sequence elements contribute to NPC binding. Moreover, it remained elusive whether different domain segments are involved in NPC binding in the context of a full-length Tpr molecule (Bangs et al., 1998).

To answer these questions, we engineered further expression vectors encoding myc-tagged Tpr deletion mutants and expressed these in PLC cells. Confocal IF microscopy revealed that only a single $\mathrm{HR}$ cluster, no. 5, located between aa 437 and 513, is essential for stable NPC binding of a recombinant Tpr protein (Figure 8). All derivatives of the $\mathrm{N}$-terminal domain that lacked this cluster of HRs generally failed to associate with NPCs. Further, all but one nearquantitatively accumulated within the nuclear interior in a detergent-extractable form (for an exception, see figure legend $8 \mathrm{~A}$, superscript 1 ).

HR cluster 5 contains 11 consecutive copies of the heptad aa consensus sequence "abcdefg" (with "a" and " $d$ " hydrophobic aa known to be critical for coiled-coil formation); arranged in tandem, this yields at least five HRs. Interestingly, one deletion mutant (Tpr 1-479) with only the first three HRs of cluster 5 was capable of stably 
A

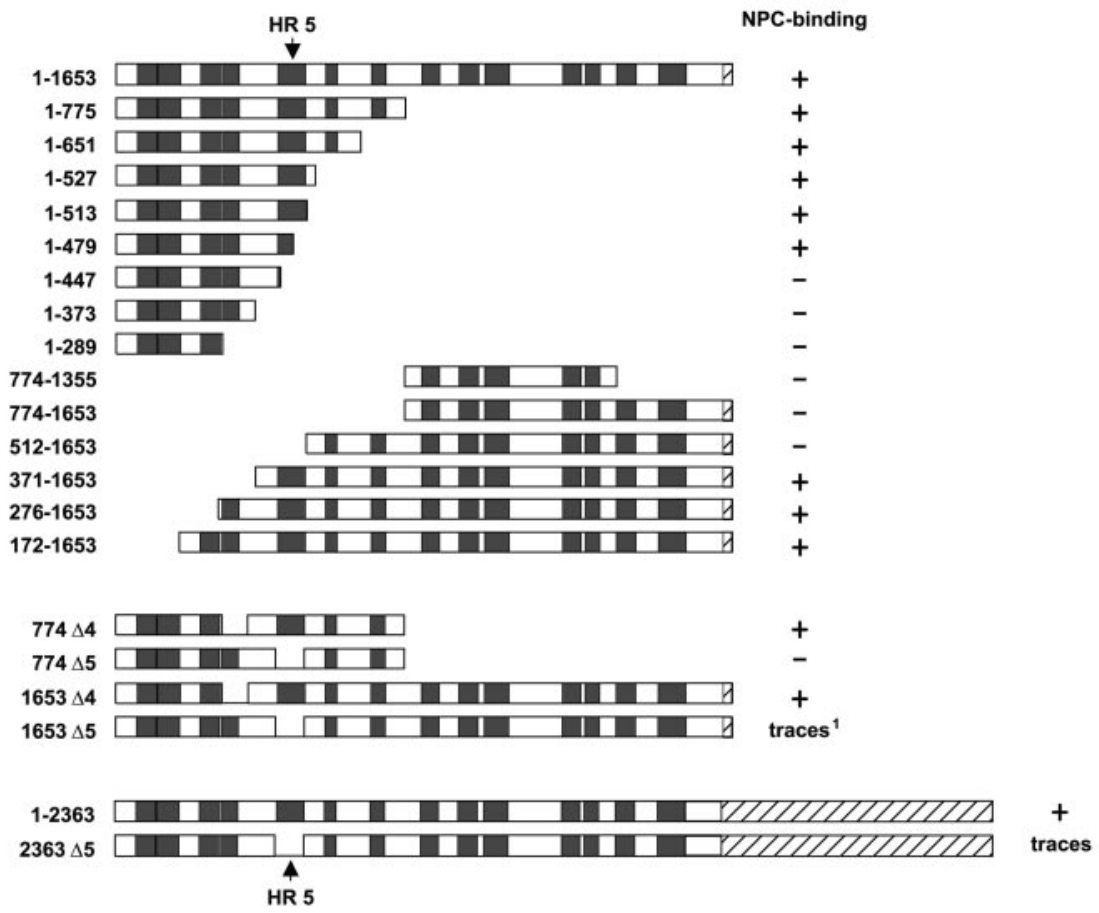

B

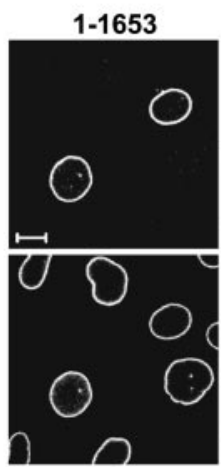

276-1653

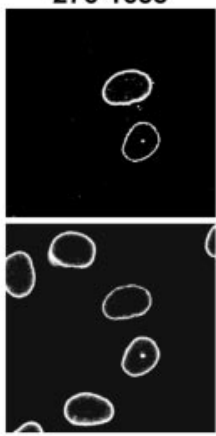

$1-479$

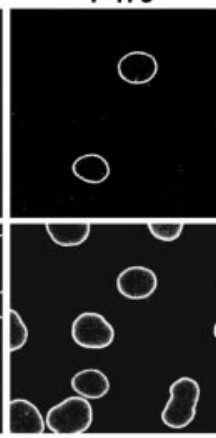

$1-1653 \Delta 4$

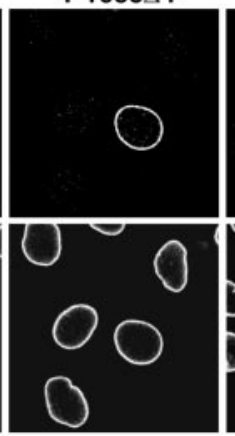

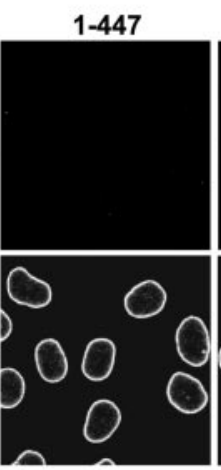

$1-1653 \Delta 5$
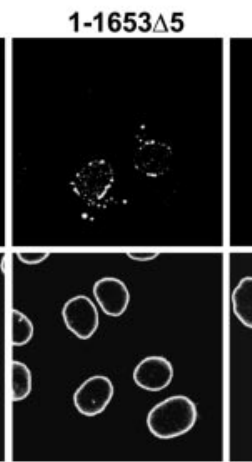

$512-1653$

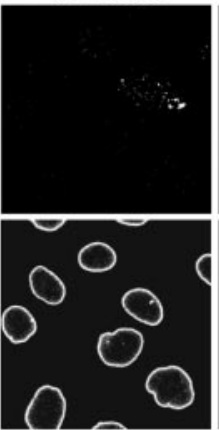

$1-2363 \Delta 5$

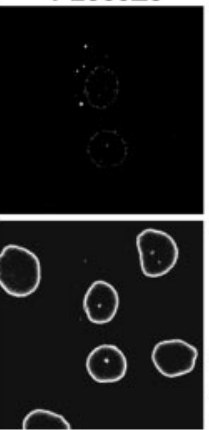

.
$371-1653$

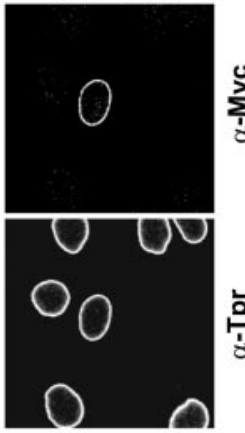

$1-774 \Delta 5$

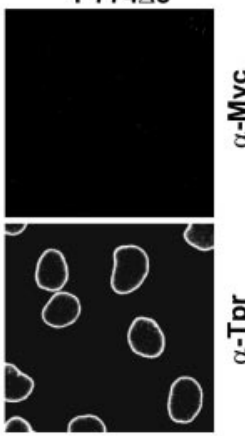

Figure 8. An N-terminal domain segment containing HR cluster 5 is essential for NPC binding of recombinant Tpr. (A) Tpr and deletion mutants encoded by

$\sum_{\Sigma}$ CMV expression vectors; each polypeptide contains a myc tag at the $\mathrm{N}$ terminus; N-terminal domain segments are also tagged with a C-terminal NLS (not shown). For constructs with internal deletions $(\Delta)$, denotations of deleted HR clusters are given. Marks indicate whether mutants can be detected at the inner nuclear periphery of transfected PLC permeabilized with Triton X-100 before fixation. Superscript 1, nuclear import of this SV40 NLS-tagged mutant was severely impaired in a majority of transfected cells, resulting in numerous detergent-stable cytoplasmic aggregates arranged along the outer nuclear membrane, but nuclear accumulation of soluble recombinant protein was obvious in cells with low expression levels. (B) Confocal IF micrographs of PLC cells transiently transfected with a selection of expression vectors referred to in A. Residual nuclear rim-staining for Tpr $1-2363 \Delta 5$ is seen only as traces and in a minor subpopulation of the transfected cells. Antibodies for double labeling are as in Figure 5. Bar, $10 \mu \mathrm{m}$, for all micrographs. binding to the NPC, whereas a deletion mutant in which two more HRs had been deleted (Tpr 1-447) failed to bind (Figure 8B).
In the context of the full-length protein, deletion of most of HR cluster 5 (Tpr 1-2363 55, lacking aa 448-510) equally caused accumulation of the mutant within the 


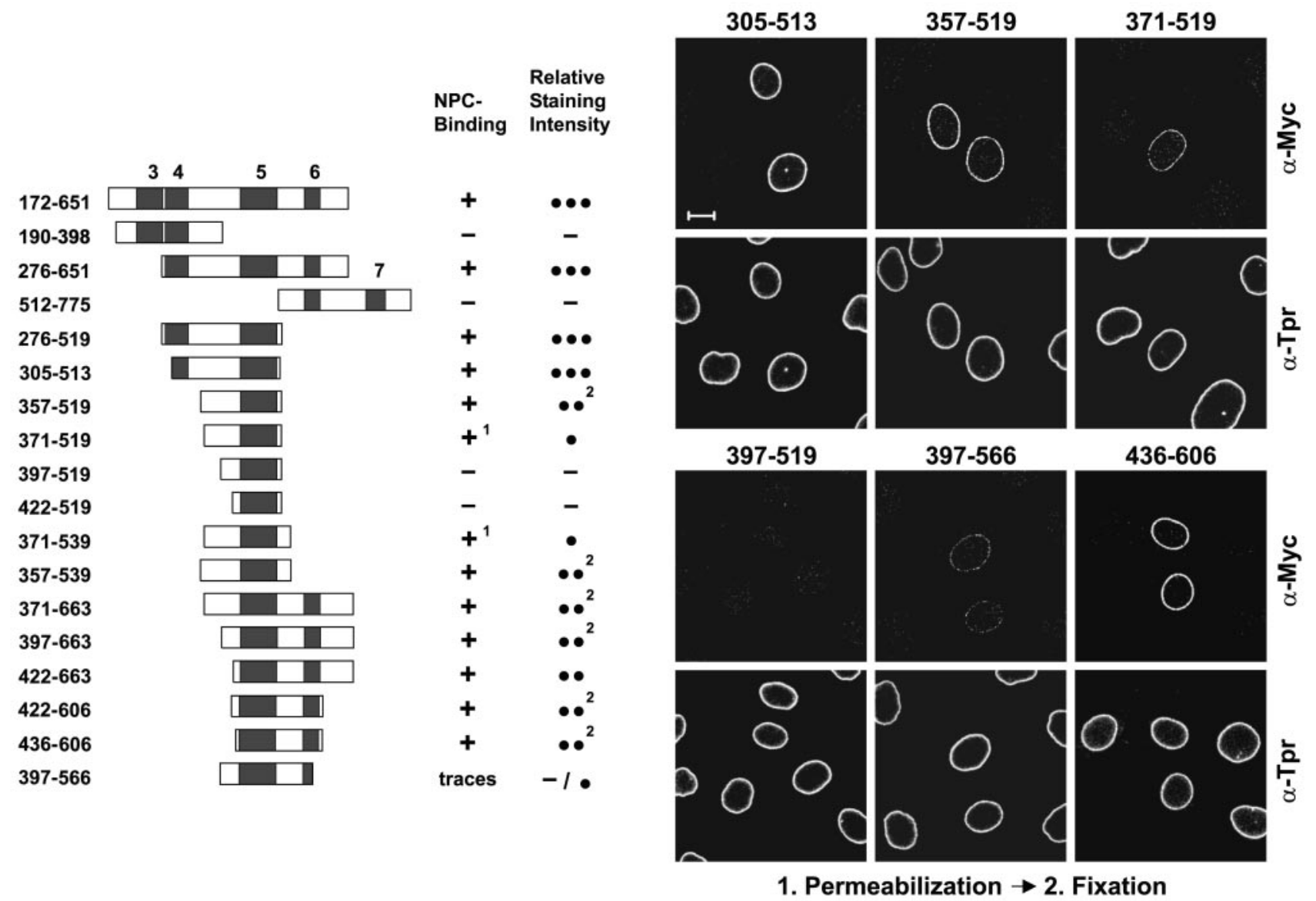

Figure 9. The smallest Tpr segment capable of NPC binding consists of HR cluster 5 and flanking sequence elements. (A) Deletion mutants encoded by CMV expression vectors, each with N-terminal myc tag and C-terminal NLS tag. First column reflects binding to the NPC in transfected PLC cells permeabilized with Triton X-100 before fixation. Relative intensity of $\alpha$-Myc immunostaining at NPCs is given in the second column (weak staining or traces thereof, one dot; moderate and intense staining, 2 and 3 dots. For rating, see MATERIALS AND METHODS). Superscript 1, after detergent extraction, the majority of these transfected cells lack NPC-bound recombinant polypeptide; superscript 2, intense nuclear rim staining only sporadically observed, i.e., in a significantly lower proportion of transfected cells than for reference construct 276-519. (B) Confocal IF micrographs of human PLC cells transiently transfected with a selection of expression vectors referred to in A. Antibodies were as for Figure 5. Same laser-scanning settings for all specimens. Bar, $10 \mu \mathrm{m}$, for all micrographs.

nuclear interior even at low expression levels (Cordes, unpublished results, but see also Figure 10). Brief detergent treatment of cells before fixation resulted in neartotal nuclear release of Tpr $1-2363 \Delta 5$, with only residual traces of nuclear rim-staining seen in a minor subpopulation of transfected cells (Figure 8B; for details of quantification, see MATERIALS AND METHODS). In contrast, occasional formation of detergent-stable cytoplasmic aggregates was still observed.

Further mapping revealed that a segment comprising only HR cluster 5 cannot bind to the NPC and that additional flanking aa sequences are required. However, these do not have to be retained on both sides of the HR cluster: short appendages of $\sim 100$ aa at either the $\mathrm{N}$ - or C-terminal side were shown to be sufficient (Figure 9).
NPC Binding, but Not General Homodimerization, Depends on Consensus Sequence Integrity of Cluster 5 HRs

To determine whether consensus sequence integrity of individual HRs in cluster 5 is essential for NPC binding, we introduced aa substitution mutations into Tpr's aa sequence. Expecting more dramatic effects with proline than with other aa substitution mutations on $\alpha$-helical structure and coiled-coil formation (O'Neil and Degrado, 1990; Chang et al., 1999, and citations therein), these were studied first. With the use of Tpr 1-774 as a model polypeptide, the substitutions were introduced into tandem repeats 2 and 4 of cluster 5 and for comparison also into flanking sequence elements and neighboring HR clusters (Table 4A). The Paircoil pro- 
gram (Berger et al., 1995) was used to help select sites where substitutions were predicted to most severely impair coiledcoil formation.

No or only minor effects were observed for proline substitutions in HR clusters 3, 4, or 6 or in HR-free segments flanking cluster 5 . In contrast, several individual mutations

Table 4. Proline and aspartic acid substitution mutations A

\begin{tabular}{|c|c|c|c|c|}
\hline $\begin{array}{l}\text { HR } \\
\text { cluster }\end{array}$ & $\begin{array}{c}\text { Amino acid } \\
\text { exchange }\end{array}$ & $\begin{array}{c}\text { Sequence segment } \\
\text { a b c de } f g a b c d e f g\end{array}$ & $\begin{array}{l}\text { NPC } \\
\text { binding }\end{array}$ & $\begin{array}{l}\text { Relative } \\
\text { Staining } \\
\text { Intensity }\end{array}$ \\
\hline none & none & & + & $\bullet$ \\
\hline 3 & $236 \mathrm{~L} \rightarrow \mathrm{P}$ & VSRLEEQMNGLKTS & + & $\bullet$ \\
\hline 3 & $257 \mathrm{~L} \rightarrow \mathrm{P}$ & VEDLLTKL P & + & $\bullet$ \\
\hline 3 & $236 P+257 P$ & $\ldots \mathrm{P} \ldots \ldots \ldots$ & + & $\bullet$ \\
\hline 4 & $310 \mathrm{~L} \rightarrow \mathrm{P}$ & VEELHKLLKEAGEA & + & $\bullet$ \\
\hline 4 & $353 \mathrm{~L} \rightarrow \mathrm{P}$ & I GRLE KE & + & $\bullet$ \\
\hline 4 & $310 P+353 P$ & $\ldots \ldots$ P.... & + & $\bullet \bullet$ \\
\hline none & $414 \mathrm{~L} \rightarrow \mathrm{P}$ & $\begin{array}{l}\text { QDQLLLEKLENKRI } \\
\ldots \ldots . . . .\end{array}$ & + & $\bullet \bullet$ \\
\hline 5 & $458 \mathrm{~L} \rightarrow \mathrm{P}$ & $\begin{array}{l}\text { VASLSVKLEQAMKF } \\
\ldots \ldots \ldots . \ldots . . .\end{array}$ & $t^{1}$ & $\bullet$ \\
\hline 5 & $459 \mathrm{E} \rightarrow \mathrm{P}$ & $\begin{array}{l}\text { VASLS VKLEQAMKF } \\
\ldots \ldots \ldots . \ldots\end{array}$ & + & $\bullet$ \\
\hline 5 & $481 \mathrm{~V} \rightarrow \mathrm{P}$ & TDKANKQSS VLERD & + & $\bullet$ \\
\hline 5 & $489 \mathrm{M} \rightarrow \mathrm{P}$ & NRRMEIQVERLLSQQ & $t^{1}$ & • \\
\hline 5 & $4911 \rightarrow P$ & NRRMEIQVESDLSQDQ & $t^{1}$ & $\bullet$ \\
\hline 5 & $458 P+489 P$ & $\ldots . P \ldots$ & $--^{2}$ & - \\
\hline none & $521 \mathrm{~V} \rightarrow \mathrm{P}$ & $\begin{array}{l}\text { HVIRDEEVSSADIS } \\
\ldots \ldots \text {. . . . . }\end{array}$ & + & $\bullet$ \\
\hline 6 & $553 \mathrm{~L} \rightarrow \mathrm{P}$ & NQRLLVALRELGET & + & $\bullet$ \\
\hline 6 & $583 \mathrm{~L} \rightarrow \mathrm{P}$ & I TELQLKLE PALTE & + & $\bullet \bullet$ \\
\hline 6 & $553 P+583 P$ & $\ldots \ldots$ P.... & + & $\bullet$ \\
\hline
\end{tabular}

\section{B}

\begin{tabular}{|c|c|c|c|c|}
\hline $\begin{array}{l}\text { HR } \\
\text { cluster }\end{array}$ & $\begin{array}{l}\text { Amino acid } \\
\text { exchange }\end{array}$ & $\begin{array}{c}\text { Sequence segment } \\
\text { a b c d e f g a b c d e f g }\end{array}$ & $\begin{array}{l}\text { NPC } \\
\text { binding }\end{array}$ & $\begin{array}{l}\text { Relative } \\
\text { Staining } \\
\text { Intensity }\end{array}$ \\
\hline 5 & $458 \mathrm{~L} \rightarrow \mathrm{D}$ & VASLSVKLEQAMKF & + & $\bullet$ \\
\hline 5 & $489 M \rightarrow D$ & NRRMEIQQVEDLSQ & + & $\bullet$ \\
\hline 5 & $491 I \rightarrow D$ & NRRMEIQQVEDLS & + & $\bullet \bullet$ \\
\hline 5 & $458 D+489 D$ & $\ldots \ldots$ D..... & $-{ }^{3}$ & $--^{3}$ \\
\hline
\end{tabular}

within HR cluster 5 impaired NPC binding, and complete abolishment was caused by double proline substitutions at L458 and M489 (Figure 10A), both in d positions of the HR motif.

Because effects resulting from introduction of two proline residues may be due to a more general structural distortion of the entire region, we also analyzed the effects of aspartic acid substitution mutations (Table 4B). Similarity in size to leucine and methionine argued against major spatial constraints for aspartic acid within the core of a coiled-coil, suggesting that an effect on NPC binding would rather be attributed to charge-induced destabilization of a (hydrophobic) coiled-coil interaction.

Whereas individual substitution of L458 or M489 had only moderate effects, the corresponding double substitution abolished the ability of Tpr 1-774 to bind to the NPC (Figure 10A). Immunoblotting of cell fractions confirmed that Tpr 1-774 with double proline or aspartic acid substitutions was present in the soluble, detergent-extractable pool, whereas the original Tpr 1-774 was largely found in the nonsoluble pellet (Hase and Cordes, unpublished data). In the context of full-length Tpr, the effect of the double aspartic acid substitution was comparable to that of HR cluster 5 deletion (Figure 8B): the mutant accumulated within the nucleus and was quantitatively extracted by brief detergent treatment; traces of residual NPC staining were observed in only a subpopulation of transfected cells (Figure 10A). Introduction of these aa substitution mutations had no obvious effect on the polypeptides' intracellular stability. In cell populations transfected with similar efficiency, recombinant $\mathrm{Tpr}$ polypeptides with and without aa substitutions were of expected approximate sizes and present in similar amounts

Table 4 (cont). Proline and charged aa substitution mutations in HRs of cluster 5 , but not within other HRs, abolish or impair binding of Tpr to the NPC. Human PLC cells were transiently transfected with expression vectors encoding the myc- and NLStagged first half of Tpr's N-terminal domain (Tpr 1-774) into which proline (A) and aspartic acid (B) substitutions had been introduced by in vitro mutagenesis. Transfected cells were permeabilized with Triton X-100 prior to fixation; NPC binding of recombinant polypeptides was studied by confocal IF microscopy. The central column shows sequence segments flanking the mutation, highlighted in blue; aa forming the "hydrophobic groove" in the original sequence are in red. Rating of relative intensity of $\alpha$-Myc immunostaining at NPCs is as in Figure 9A. Superscript 1, in the context of Tpr 1-774, some proline substitutions in HR cluster 5 cause additional formation of multiple intranuclear aggregates of small, dotlike appearance in 10-35\% of transfected cells. Superscript 2, double proline substitution for L458/M489 in the context of Tpr 1-1653 has similar effects as described for Tpr 1-1653 55 (Figure 8A), i.e., significantly impaired nuclear import of the mutant, and formation of numerous cytoplasmic aggregates at the outer nuclear membrane; similar, but less severe import defects are also noted for Tpr 1-1653 with individual proline or double aspartic acid substitutions for L458/M489. Accumulation of aggregates at the nuclear periphery is NLS dependent; upon removal of the NLS tag, mutants remain randomly distributed within the cytoplasm. Superscript 3, in the context of Tpr 1-774, double substitution of L458/M489 for aspartic acid completely abolishes NPC binding; in the context of Tpr 1-2363, weak NPC-staining can be seen in a minor subpopulation of transfected cells. 
Figure 10. Double aa substitution mutations within HR cluster 5 abolish NPC binding but have no obvious effect on intracellular stability. (A) Confocal IF micrographs of PLC cells transiently transfected with a selection of expression vectors encoding Tpr polypeptides with double aa substitutions in HR cluster 5. Cells were permeabilized with Triton X-100 after or before fixation. Construct Tpr 1-774, with original L458 and M489, is shown as reference. Antibodies were as for Figure 5. Note that Tpr polypeptides with double proline or charged aa substitutions of aa 458 and 489 do not bind to NPCs but accumulate within the nuclear interior (top). This intranuclear pool of Tpr mutants is quantitatively released by brief permeabilization of cells before fixation (bottom). Residual nuclear rim-staining for Tpr 1-2363 with double aa substitutions in HR cluster 5 is seen only as traces and in a minor subpopulation of the transfected cells. Same laser-scanning settings for all specimens. Bar, $10 \mu \mathrm{m}$, for all micrographs. (B) Immunoblot detection of recombinant Tpr polypeptides with and without aa substitutions. Each lane contains equal amounts of total protein from cells transfected with different Tpr expression vector constructs or empty vector alone (mock). Cells on culture dishes were directly solubilized in boiling sample buffer. In parallel, transfection efficiency for the different full-length constructs (right) or for constructs encoding truncated forms of recombinant Tpr was controlled by IF microscopy to be within the same order of magnitude $(\Delta<40 \%)$. Slightly decreased electrophoretic mobility of Tpr 1-774 with double proline substitutions, relative to other Tpr 1-774 variants, was repeatedly observed. Myc-tagged polypeptides were detected by $\mathrm{mAb}$ 9E10.
A

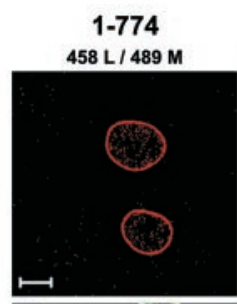

1-774 $458 \mathrm{~L} \rightarrow P / 489 M \rightarrow$
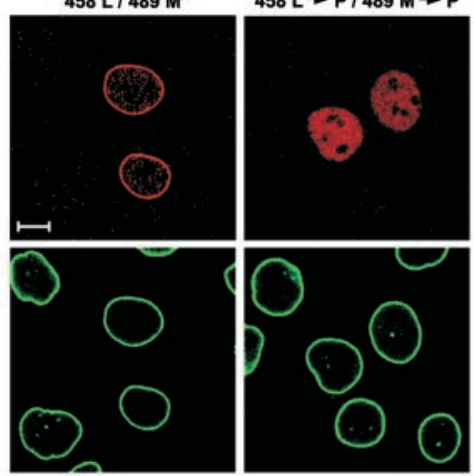

1. Fixation $\rightarrow 2$. Permeabilization
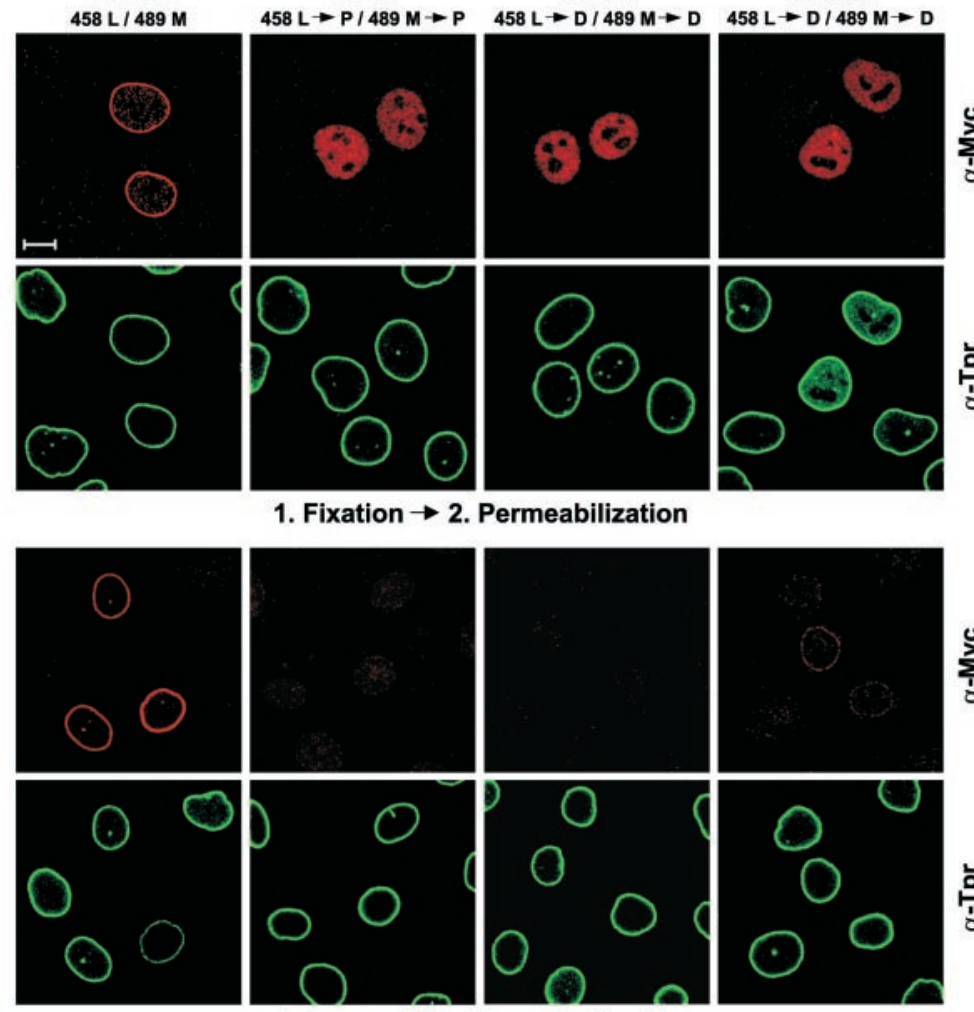

1. Permeabilization $\rightarrow 2$. Fixation

B

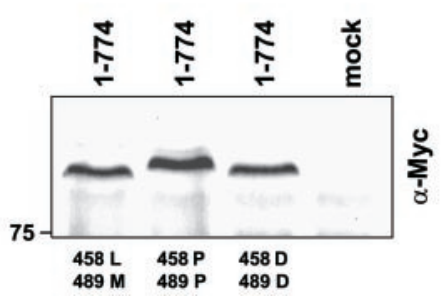

1-2363

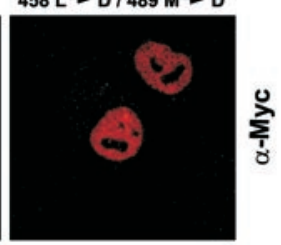

$\frac{\text { 흥 }}{8}$
$458 \mathrm{~L} \rightarrow \mathrm{D} / 489 \mathrm{M} \rightarrow \mathrm{D}$
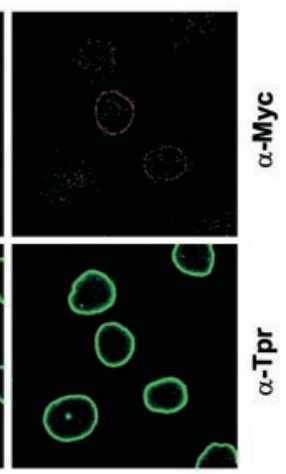

$\frac{2}{\frac{2}{6}}$

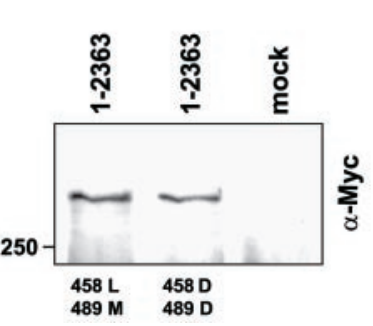

as controlled by immunoblotting of total cell proteins (Figure 10B).

In contrast to NPC binding, Tpr's general ability to homodimerize, as monitored with the yeast two-hybrid system with the use of Tpr model segments 1-774 and 172-651, was not abrogated. Double substitution mutations did not abolish homodimerization between pairs of monomeric mutants or between a monomeric mutant and the corresponding mutation-free polypeptide (Figure 11).

\section{DISCUSSION}

In this study we have followed up on ideas that suggest that Tpr represents a filament-forming, architectural element of the nuclear interior. In fact, in amphibian oocytes, Tpr had been found associated with NPC-attached filaments projecting into the nuclear interior (Cordes et al., 1997), and in different types of Drosophila cells, Tpr had been localized throughout the extrachromosomal nuclear interior where it had been suggested to form or be part of a filamentous intranuclear skeleton (Zimowska et al., 1997). Moreover, in transfected hamster cells studied by IF microscopy, small recombinant segments of human Tpr have been reported to occasionally form striking filamentous arrays extending throughout the cytoplasm (Bangs et al., 1998), suggesting that Tpr may be capable of homopolymerization in vivo. However, the only unequivocal evidence that some regions of Tpr may engage in homodimeric interactions came from the finding that the short Tpr segment (aa 1-142) present in the Tpr-Met fusion protein (Park et al., 1986) is involved in dimerization of this chimeric oncogene product (Rodrigues et al., 1993).

To gain insight into Tpr's ultrastructural properties, we have studied bacterially synthesized Tpr segments by CD 


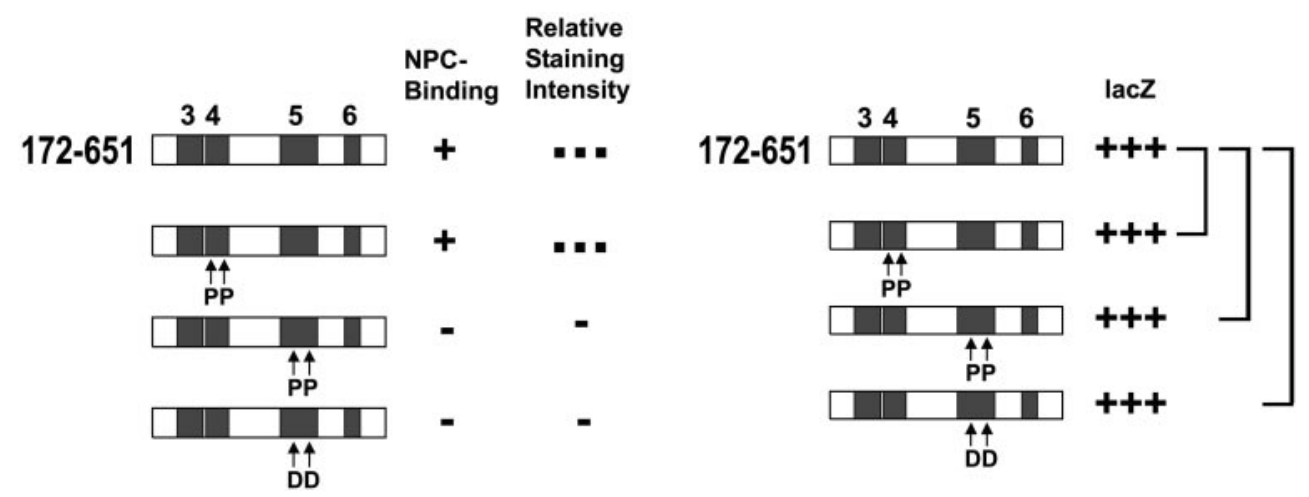

Figure 11. Double aa substitution mutations within HR cluster 5 do not abolish Tpr's general ability to homodimerize. Selection of experiments in which effects on NPC binding and homodimerization were compared. Double aa substitutions were introduced into mycand NLS-tagged Tpr model segment 172-651 (assessment of NPC binding in transfected PLC cells as in Figure 9) and into BD and AD fusions of the same segment. Two-hybrid interactions were studied between identical segments and between mutation-free segment and double aa substitution variants (brackets). Rating of lacZ reporter gene expression is as in Figure 4.

spectroscopy, EM, and chemical cross-linking. The results directly reflect the bipartite structure of Tpr, demonstrate its ability to form homodimers, and show that polypeptides of the N-terminal domain are dominated by $\alpha$-helices arranged in a double-stranded coiled coil of rod-like shape. Interestingly, the average rod length of $51 \mathrm{~nm}$ measured for Tpr polypeptide 1-398 was close to a maximal theoretical value of $59 \mathrm{~nm}$ calculated for an entirely extended molecule of exclusively $\alpha$-helical conformation with $1.485 \AA$ per helical residue. When considering only the predicted $\alpha$-helical regions of the Tpr segment, theoretical lengths range from 50.8 to $53.5 \mathrm{~nm}$. For the other N-terminal segment, Tpr 774-1370, the theoretical maximal, and $\alpha$-helical lengths were 88 and 75-80 nm, respectively. Extrapolation of these values would yield a theoretical length between 169 and $208 \mathrm{~nm}$ for a full-length $\mathrm{N}$-terminal domain of entirely rectilinear structure. However, in contrast to Tpr 1-398 of rather uniform rectilinear appearance, actual Tpr 774-1370 rods not only varied significantly in length but also appeared to be of more flexible shape. Whether this may point to a more flexible region within the full-length protein remains to be investigated once Tpr or its full-length $\mathrm{N}$-terminal domain have been purified. Pentapeptide sequences GKGQP at aa 924928 and GVQGP at aa 1177-1181 represent candidate regions that may cause natural hinges in Tpr's rod domain. Rectilinearity of Tpr may also be interrupted by a cluster of five proline residues located between aa 642 and 658. Similarly, rod-shaped molecules seen in protein fractions enriched for recombinant yeast Mlp1 were reported to be very heterogeneous in appearance when studied by EM (Kosova et al., 2000).

A yeast two-hybrid approach complemented our analysis of Tpr's ultrastructural properties and revealed that the two strands of the homodimer are arranged in parallel and in register. Interestingly, very high levels of reporter gene activity were observed after homodimerization of $\mathrm{Tpr}$ polypeptide 1-235 and Tpr 774-1178: the one including the segment involved in dimerization of the Tpr-Met fusion protein and the other a sequence motif (aa 1004-1013) shown to facilitate formation of other two-stranded coiled coils (Kammerer et al., 1998). However, whether these regions may reflect preferential assembly sites for Tpr monomers remains uncertain because yeast reporter gene activity is not necessarily equally proportional to the actual interac- tions between different pairs of fusion proteins. It can be modulated by various factors, including fusion protein stability and nuclear import and sterical constraints (Ye and Worman, 1995; Ayers et al., 1999).

To investigate whether full-length Tpr, or any of its segments, may self-assemble into homopolymeric filaments in vivo, we expressed these polypeptides in cultured human cells and studied them by confocal IF microscopy, cell fractionation, and immuno-EM. In the sequel, none of the Tpr polypeptides were found to form structures of marked filamentous appearance. In this respect, Tpr is similar to other nonpolymerizing coiled-coil-dominated proteins of large size, such as different members of the golgin family, which in terms of degree of sequence identity are closely related to Tpr (Misumi et al., 1997; Kjer-Nielsen et al., 1999). However, Tpr clearly differs from several other coiled-coil proteins, which upon overexpression have the potential to readily form spectacular filament bundles even in the nuclear interior of somatic cells (Bader et al., 1991; Bridger et al., 1998; Yuan et al., 1998). It remains to be investigated whether origin of cytoplasmic filament bundles in hamster BHK cells, IF labeled with antibodies reactive for recombinant segments of hTpr (Bangs et al., 1998), may be explained differently than by self-assembly of Tpr molecules.

Here, we show that surplus Tpr, which does not bind to the NPC, remains either in a soluble form or assembles into aggregates of entangled molecules. Rectilinear filament bundles or paracrystalline structures as part of such aggregates, and seen upon overexpression of other coiled-coil proteins (Saredi et al., 1996; Klapper et al., 1997), were not detected. Standard transmission EM analysis of ultrathin sections, however, did not allow exclusion of the possibility that more delicate, filigree-like regular assemblies of Tpr molecules may underlie these aggregates. To address this question, electron tomography with the COMET software technology (Miralles et al., 2000) can be utilized to study these structures at $\sim 3 \mathrm{~nm}$ of resolution in three dimensions.

In the nucleus, recombinant full-length Tpr accumulated at the nuclear periphery in a zone corresponding or slightly distal to the location of the nuclear basket's terminal ring. Although orientation and spatial arrangement of Tpr in relation to the basket remains a topic for future studies, the data presented here clearly demonstrate the abrupt transition between this NPC-proximal and the intranuclear pool 
of recombinant Tpr. This further argues against the idea that a surplus of Tpr may lead to "growth" of homopolymeric filaments toward the nuclear interior starting at NPCs as nucleation sites.

Furthermore, recombinant Tpr deep within the nucleus was not found stably associated with any specific intranuclear structure. Occasional dot-like IF staining with Tpr antibodies in the nuclear interior of PLC cells (Figures 5 and $8-10)$ was found by serial confocal sectioning to mostly represent funnel-like invaginations of the nuclear envelope (see also Fricker et al., 1997) also reactive with antibodies for lamins and other NPC proteins such as Nup358/RanBP2 (Cordes, unpublished data). In an earlier investigation, we had occasionally observed dot-like Tpr staining proximal to the nucleoli of cultured cells that had been fixed with coagulating agents such as methanol/acetone (Cordes et al., 1997; see also Zimowska et al., 1997). Whether this may reflect a fixation artifact due to coagulation of soluble Tpr or a problem of resolution due to specimen dehydration and shrinkage along the z-axis (Bacallao et al., 1995, and citations therein) remains to be investigated. In the present study, in which cross-linkers had been used for fixation, this type of intranuclear staining was not observed independent of whether antibodies specific for Tpr's N- or C-terminal domain were used. It appears unlikely that treatment with FA should result in simultaneous masking of several different Tpr epitopes only when the protein would occur bound to intranuclear structures but not when attached to the NPC.

In contrast, the intranuclear pool of recombinant Tpr was found to represent a monodisperse, soluble form of $7.5 \mathrm{~S}$, similar to a soluble pool of wild-type Tpr that represents $<5 \%$ of the total amount of Tpr in an interphase cell population. This endogenous pool may reflect on-going synthesis of wild-type Tpr for which there may be a demand concomitant to the doubling of NPC numbers during cell cycle progression from G1 to G2 (Maul, 1977). However, whether soluble forms of wild-type Tpr may also fulfill functions different from those at the nuclear periphery, and whether marked intranuclear occurrence of Tpr in insect salivary gland cells (Zimowska et al., 1997; Fomproix and Cordes, unpublished data) represents similar soluble pools, remains to be investigated.

A low sedimentation coefficient but high Stokes radius for soluble Tpr was not a surprise, expecting a highly elongated molecule. The calculated molecular weight of $\sim 509,000$ indicates that Tpr in its soluble form is likely to occur as a homodimer. Soluble forms of other large-sized coiled-coil proteins are also known to have low sedimentation coefficients compared with those of globular proteins of similar size (Cantor and Schimmel, 1980). Homodimers of mammalian giantin (macrogolgin), a member of golgin subfamily b with a molecular mass of $376 \mathrm{kDa}$ for the monomer (Seelig et al., 1994; Sohda et al., 1994), were shown to have a sedimentation coefficient of only 9 S (Linstedt and Hauri, 1993). Mammalian myosins of the class II subfamily represent further examples: composed of two pairs of light chains of $17-24 \mathrm{kDa}$, plus two heavy chains of $\sim 220-230 \mathrm{kDa}$ which form a homodimeric coiled-coil rod, these assemblies can occur in a highly extended conformation, with rectilinear rod domains of up to $160 \mathrm{~nm}$ in length and a Stokes radius of $185 \AA$ (Nozaki et al., 1976; Elliott and Offer, 1978). Total mass of soluble myosin II complexes can exceed $500 \mathrm{kDa}$, whereas corresponding sedimentation coefficients can be as low as 6 S (Trybus et al., 1982; Takashima et al., 1988).

To complement our analysis of Tpr's quaternary structural properties, we have mapped the sequence elements essential for binding to the NPC and show that such binding depends on sequence integrity of HR cluster 5. At present, we cannot exclude, but consider it unlikely, that the cluster 5 region represents a site essential for homo-oligomerization between Tpr dimers. In such a scenario, mutation of HR cluster 5 would still allow homodimerization between recombinant molecules but not the homo-oligomeric binding to wild-type Tpr dimers attached to the NPC. As the alternative, we propose that Tpr binds to the NPC as a dimer in which the HR cluster 5 region represents the direct binding site for one or several NPC proteins. This binding may occur via heteromeric coiled-coil interaction between the HRs of Tpr and those of a binding partner. However, a homodimeric cluster 5 coiled coil as a structural unit may be recognized by the binding partner as well. In this latter case, the binding partner would not have to be a coiled-coil protein itself. Interestingly, a short Tpr segment (1-479), making up only the first half of HR cluster 5, was capable of binding to the NPC, whereas aa substitutions in both the first and second half were required to abolish NPC binding, indicative that both may include individual binding modules. However, a segment solely consisting of HR cluster 5 cannot bind to the NPC but requires short flanking segments at either its $\mathrm{N}$ - or $\mathrm{C}$-terminal side. These may be required for structural stabilization of HR cluster 5 or may represent part of the binding site itself. The finding that they do not have to be present on both sides of the HR cluster now explains seemingly conflicting observations between earlier studies (Bangs et al., 1998; Cordes et al., 1998).

Future investigations may reveal whether Tpr's NPCbinding domain is a target region for mechanisms regulating the binding and release of Tpr to and from NPCs in interphase and prophase, respectively (Byrd et al., 1994). This may include posttranslational modification of the binding domain. Regulated masking by non-NPC proteins may represent an alternative means to prevent NPC binding. Future studies may also impart whether mutations that affect Tpr's binding to the NPC can also occur naturally in living organisms and whether these provoke pathological effects.

Provided that human Tpr in somatic cells does not selfassemble into homopolymeric filaments of marked length, this raises the question as to the composition and assembly of the extensive, NPC-attached filament bundles in amphibian oocytes (Ris and Malecki, 1993; Ris, 1997). To these, not only Tpr, but also at least one other NPC protein, Nup153 (Sukegawa and Blobel, 1993), have been found associated (Cordes et al., 1993). However, in the amphibian oocyte high amounts of proteins of the NPC proper, considered to be later required for embryogenesis, are stockpiled in vast numbers of cytoplasmic annulate lamellae (Cordes et al., 1995), whereas several proteins specific for the NPC's nucleoplasmic side, such as Tpr, Nup153, and others, are either absent or present only in substoichiometric amounts (Cordes et al., 1997; Cordes, unpublished results). These karyophilic NPC proteins may instead be stockpiled within the oocyte's nucleus where they coassemble and in such a way jointly form the NPC-attached filamentous structures characteristic for this particular cell type. 
On the other hand, realization that Tpr is tightly associated with the NPC and cannot self-assemble into stable filaments or geodesic networks traversing the nuclear interior of somatic cells argues against a universal role as homopolymeric architectural element of an extensive fibrillar nucleoskeleton. Recent results also do not support the idea that Tpr may function as a central backbone component of the nuclear basket: NPC reassembly after mitosis, including the reincorporation of nuclear basket proteins such as Nup153, and recovery of nuclear transport activity clearly precedes late reassociation of Tpr to the NPC (Bodoor et al., 1999; Haraguchi et al., 2000; see also Byrd et al., 1994). This is also in agreement with immuno-EM localization of Tpr slightly distal to the nuclear basket's terminal ring.

Alternatively, a role in subnuclear chromatin organization as shown for the yeast Tpr homologue Mlp2 (Galy et al., 2000) may also outline a possible function of the mammalian counterpart. In a more static setting, NPC-associated homodimers of human Tpr may represent an anchor site for perinuclear chromatin (Cordes et al., 1997), perhaps by virtue of the C-terminal domains placed in position by a rather flexible Tpr rod acting as a swivel arm. In another scenario, Tpr may serve as local operational platform for proteins with more dynamic roles in perinuclear organization and other nuclear processes.

\section{ACKNOWLEDGMENTS}

We thank Drs. Astrid Gräslund, Eberhard Spiess, Werner Franke, Bertil Daneholt, Örjan Wrange, and Björn Öbrink for the opportunity to use equipment in their laboratories. Furthermore, we gratefully acknowledge the help and advice of Dr. Jüri Jarvet in the use of CD spectroscopy, Dr. Spiess in heavy metal rotary shadowing, and Kristmundur Sigmundsson in gel filtration chromatography. Special thanks go to Sonja Reidenbach for assistance in ultrathin sectioning of EM specimen. We also wish to thank Kyle Sousa and Drs. Georg Krohne, Piergiorgio Percipalle, and David Bear for critical reading of the manuscript in whole or in part. We further acknowledge the help of Irmgard Cordes with the graphical work and of Drs. Ulf Skoglund, Lars-Göran Öfverstedt, Sergej Masich, and Vadim Frolov with different computer programs. Thanks also to Norbert Mücke and Drs. Skoglund, Harald Herrmann, Ozan Öktem, and Johan Thyberg for helpful suggestions. Confocal microscopy was performed on a Zeiss LSM 510 purchased by a grant from the Ingabritt and Arne Lundbergs Research Foundation to Dr. Öbrink. This investigation was supported by grants to V.C.C. from the Swedish Natural Research Council, the Marianne and Marcus Wallenberg Foundation, the foundation Cancerföreningen i Stockholm, and the Human Frontier Science Program.

\section{REFERENCES}

Arlucea, J., Andrade, R., Alonso, R., and Arechaga, J. (1998). The nuclear basket of the nuclear pore complex is part of a higher-order filamentous network that is related to chromatin. J. Struct. Biol. 124, $51-58$.

Ayers, N.A., Wilkinson, D.A., Fitzgerald, T.J., and Carlson, G.M. (1999). Self-association of the $\alpha$ subunit of phosphorylase kinase as determined by two-hybrid screening. J. Biol. Chem. 274, 3558335590.

Bacallao, R., Kiai, K., and Jesaitis, L. (1995). Guiding principles of specimen preservation for confocal fluorescence microscopy. In: Handbook of Biological Confocal Microscopy, J.B. Pawley, ed., New York: Plenum Press, 311-325.
Bader, B.L., Magin, T.M., Freudenmann, M., Stumpp, S., and Franke, W.W. (1991). Intermediate filaments formed de novo from tail-less cytokeratins in the cytoplasm and in the nucleus. J. Cell Biol. 115, 1293-1307.

Bangs, P.L., Burke, B., Powers, C., Graig, R., Purohit, A., and Doxsey, S. (1998). Functional analysis of Tpr: identification of nuclear pore complex association and nuclear localization domains and a role in mRNA export. J. Cell Biol. 143, 1801-1812.

Berger, B., Wilson, D.B., Wolf, E., Tonchev, T., Milla, M., and Kim, P.S. (1995). Predicting coiled coils by use of pairwise residue correlations. Proc. Natl. Acad. Sci. USA 92, 8259-8262.

Blum, H., Beier, H., and Gross, H.J. (1987). Improved silver staining of plant proteins, RNA and DNA in polyacrylamide gels. Electrophoresis 8, 93-99.

Bodoor, K., Shaikh, S., Salina, D., Raharjo, W.H., Bastos, R., Lohka, M., and Burke, B. (1999). Sequential recruitment of NPC proteins to the nuclear periphery at the end of mitosis. J. Cell Sci. 112, 22532264.

Bridger, J.M., Hermann, H., Münkel, C., and Lichter, P. (1998). Identification of an interchromosomal compartment by polymerization of nuclear-targeted vimentin. J. Cell Sci. 111, 1241-1253.

Byrd, D.A., Sweet, D.J., Pante, N., Konstantinov, K.N., Guan, T., Saphire, A.C.S., Mitchell, P.J., Cooper, C.S., Aebi, U., and Gerace, L. (1994). Tpr, a large coiled coil protein whose amino terminus is involved in activation of oncogenic kinases, is localized to the cytoplasmic surface of the nuclear pore complex. J. Cell Biol. 127, 1515-1526.

Cantor, C.R., and Schimmel, P.R. (1980). Biophysical Chemistry. II. Techniques for the Study of Biological Structure and Function. San Fransisco, CA: W.H. Freeman.

Carpenter, D.A., and Ip, W. (1996). Neurofilament triplet protein interactions: evidence for the preferred formation of NF-L-containing dimers and a putative function for the end domains. J. Cell Sci. 109, 2493-2498.

Chang, D.K., Cheng, S.F., Trivedi, V.D., and Lin, K.L. (1999). Proline affects oligomerization of a coiled coil by inducing a kink in a long helix. J. Struct. Biol. 128, 270-279.

Cordes, V.C., Hase, M.E., and Müller, L. (1998). Molecular segments of protein Tpr that confer nuclear targeting and association with the nuclear pore complex. Exp. Cell. Res. 245, 43-56.

Cordes, V.C., Reidenbach, S., and Franke, W.W. (1995). High content of a nuclear pore complex protein in cytoplasmic annulate lamellae of Xenopus oocytes. Eur. J. Cell Biol. 68, 240-255.

Cordes, V.C., Reidenbach, S., Köhler, A., Stuurman, N., van Driel, R., and Franke, W.W. (1993). Intranuclear filaments containing a nuclear pore complex protein. J. Cell Biol. 123, 1333-1344.

Cordes, V.C., Reidenbach, S., Rackwitz, H.-R., and Franke, W.W. (1997). Identification of protein $\mathrm{p} 270 / \mathrm{Tpr}$ as a constitutive component of the nuclear pore complex-attached intranuclear filaments. J. Cell Biol. 136, 515-529.

Elliott, A., and Offer, G. (1978). Shape and flexibility of the myosin molecule. J. Mol. Biol. 123, 505-519.

Fields, S., and Song, O.-K. (1989). A novel genetic system to detect protein-protein interactions. Nature 340, 245-246.

Franke, W.W., and Scheer, U. (1970). The ultrastructure of the nuclear envelope of amphibian oocytes: a reinvestigation. II. The immature oocyte and dynamic aspects. J. Ultrastruct. Res. 30, 317-327.

Fricker, M., Hollinshead, M., White, N., and Vaux, D. (1997). Interphase nuclei of many mammalian cell types contain deep, dynamic, tubular membrane-bound invaginations of the nuclear envelope. J. Cell Biol. 136, 531-544. 
Frishman, D., and Argos, P. (1997). Seventy-five percent accuracy in protein secondary structure prediction. Proteins 27, 329-335.

Galy, V., Olivo-Marin, J.-C., Scherthan, H., Doye, V., Rascalou, N., and Nehrbass, U. (2000). Nuclear pore complexes in the organization of silent telomeric chromatin. Nature 403, 108-112.

Gant, T.M., and Wilson, K.L. (1997). Nuclear assembly. Annu. Rev. Cell Dev. Biol. 13, 669-695.

Goldberg, M.W., and Allen, T.D. (1992). High resolution scanning electron microscopy of the nuclear envelope: demonstration of a new, regular, fibrous lattice attached to the baskets of the nucleoplasmic face of the nuclear pores. J. Cell Biol. 119, 1429-1440.

Greco, A., Pierotti, M.A., Bongarzone, I., Pagliardini, S., Lanzi, C., and Della Porta, G. (1992). TRK-T1 is a novel oncogene formed by the fusion of TPR and TRK genes in human papillary thyroid carcinoma. Oncogene 7, 237-242.

Gueth-Hallonet, C., Wang, J., Harboth, J., Weber, K., and Osborn, M. (1998). Induction of a regular nuclear lattice by overexpression of NuMA. Exp. Cell Res. 243, 434-452.

Handbook of Biochemistry (1970). 2nd ed., H.A. Sober, ed., Cleveland, $\mathrm{OH}$ : Chemical Rubber Publishing.

Haraguchi, T., Koujin, T., Hayakawa, T., Kaneda, T., Tsutsumi, C., Imamoto, N., Akazawa, C., Sukegawa, J., Yoneda, Y., and Hiraoka, Y. (2000). Live fluorescence imaging reveals early recruitment of emerin, LBR, RanBP2, and Nup153 to reforming functional nuclear envelopes. J. Cell Sci. 113, 779-794.

Herrmann, H., and Lichter, P. (1999). New ways to look at the interchromosomal-domain compartment. Protoplasma 209, 157-165.

Ishikawa, F., Takaku, F., Nagao, M., and Sugimura, T. (1987). Rat c-raf oncogene activation by a rearrangement that produces a fused protein. Mol. Cell. Biol. 7, 1226-1232.

Jarnik, M., and Aebi, U. (1991). Towards a more complete 3 Dstructure of the nuclear pore complex. J. Struct. Biol. 107, 291-308.

Johnson, W.C. (1990). Protein secondary structure and circular dichroism: a practical guide. Proteins 7, 205-214.

Kammerer, R.A., Schulthess, T., Landwehr, R., Lustig, A., Engel, J., Aebi, U., and Steinmetz, M.O. (1998). An autonomous folding unit mediates the assembly of two-stranded coiled coils. Proc. Natl. Acad. Sci. USA 95, 13419-13424.

Kjer-Nielsen, L., van Vliet, C., Erlich, R., Toh, B.-H., and Gleeson, P.A. (1999). The Golgi-targeting sequence of the peripheral membrane protein p230. J. Cell Sci. 112, 1645-1654.

Kosova, B., Pante, N., Rollenhagen, C., Podtelejnikov, A., Mann, M., Aebi, U., and Hurt, E. (2000). Mlp2p, a component of nuclear pore attached intranuclear filaments, associates with Nic96p. J. Biol. Chem. 275, 343-350.

Klapper, M., Exner, K., Kempf, A., Gehrig, C., Stuurman, N., Fisher, P., and Krohne, G. (1997). Assembly of A- and B-type lamins studied in vivo with the baculovirus system. J. Cell Sci. 110, 2519-2532.

Laskey, L.A. (2000). The nucleoskeleton go EAST, young man. Nat. Cell Biol. 2, E74-E76.

Leung, C.L., and Liem, R.K.H. (1996). Characterization of interactions between the neurofilament triplet proteins by the yeast twohybrid system. J. Biol. Chem. 271, 14041-14044.

Linstedt, A.D., and Hauri, H-P. (1993). Giantin, a novel conserved Golgi membrane protein containing a cytoplasmic domain of at least $350 \mathrm{kDa}$. Mol. Biol. Cell 4, 679-693.

Lupas, A. (1996). Coiled coils: new structures and new functions. Trends Biochem. Sci. 21, 375-382.
Maul, G. (1977). The nuclear and the cytoplasmic pore complex: structure, dynamics, distribution, and evolution. Int. Rev. Cytol. Suppl. 6, 75-186.

Meng, J., Khan, S., and Ip, W. (1996). Intermediate filament domain interactions as revealed by two-hybrid screens. J. Biol. Chem. 271, $1599-1604$.

Miralles, F., Öfverstedt, L.-G., Sabri, N., Aissouni, Y., Hellman, U., Skoglund, U., and Visa, N. (2000). Electron tomography reveals posttranscriptional binding of pre-mRNPs to specific fibers in the nucleoplasm. J. Cell Biol. 148, 271-282.

Misumi, Y., Sohda, M., Yano, A., Fujiwara, T., and Ikehara, Y. (1997). Molecular characterization of GCP170, a 170-kDa protein associated with the cytoplasmic face of the Golgi membrane. J. Biol. Chem. 272, 23851-23858.

Mitchell, P.J., and Cooper, C.S. (1992). The human tpr gene encodes a protein of 2094 amino acids that has extensive coiled-coil regions and an acidic C-terminal domain. Oncogene 7, 2329-2333.

Newman, J.R.S., Wolf, E., and Kim, P.S. (2000). A computationally directed screen identifying interacting coiled coils from Saccharomyces cerevisiae. Proc. Natl. Acad. Sci. USA 97, 13203-13208.

Nozaki, Y., Schechter, N.M., Reynolds, J.A., and Tanford, C. (1976). Use of gel chromatography for the determination of the Stokes radii of proteins in the presence and absence of detergents: a reexamination. Biochemistry 15, 3884-3890.

Ohno, M., Fornerod, M., and Mattaj, I.W. (1998). Nucleocytoplasmic transport: the last 200 nanometers. Cell 92, 327-336.

O'Neil, K.T., and Degrado, W.F. (1990). A thermodynamic scale for the helix-forming tendencies of the commonly occurring amino acids. Science 250, 646-651.

Paddy, M.R. (1998). The Tpr protein: linking structure and function in the nuclear interior? Am. J. Hum. Genet. 63, 305-310.

Park, M., Dean, M., Cooper, C.S., Schmidt, M., O’Brien, S.J., Blair, D.G., and Woude, G.F.V. (1986). Mechanism of met oncogene activation. Cell 45, 895-904.

Pemberton, L.F., Blobel, G., and Rosenblum, J.S. (1998). Transport routes through the nuclear pore complex. Curr. Opin. Cell Biol. 10, 392-399.

Pesheva, P., Spiess, E., and Schachner, M. (1989). J1-160 and J1-180 are oligodendrocyte-secreted non-permissive substrates for cell adhesion. J. Cell Biol. 109, 1765-1778.

Pharmacia (1998). Handbook of Gel Filtration: Principles and Methods, 8th ed., Piscataway, NJ: Pharmacia.

Ris, H. (1989). Three-dimensional imaging of cell ultrastructure with high resolution low voltage SEM. Inst. Phys. Conf. Ser. 98, 657-662.

Ris, H. (1991). The three-dimensional structure of the nuclear pore complex as seen by high voltage electron microscopy and high resolution low voltage scanning electron microscopy. EMSA Bull. $21,54-56$.

Ris, H. (1997). High-resolution field-emission scanning electron microscopy of nuclear pore complex. Scanning 19, 368-375.

Ris, H., and Malecki, M. (1993). High-resolution field emission scanning electron microscope imaging of internal cell structures after epon extraction from sections: a new approach to correlative ultrastructural and immunocytochemical studies. J. Struct. Biol. 111, 148-157.

Rodrigues, G.A., and Park, M. (1993). Dimerization mediated through a leucine zipper activates the oncogenic potential of the met receptor tyrosine kinase. Mol. Cell. Biol. 13, 6711-6722.

Ryan, K.J., and Wente, S.R. (2000). The nuclear pore complex: a protein machine bridging the nucleus and cytoplasm. Curr. Opin. Cell Biol. 12, 361-371. 
Saredi, A., Howard, L., and Compton, D.A. (1996). NuMA assembles into an extensive filamentous structure when expressed in the cell cytoplasm. J. Cell Sci. 109, 619-630.

Scheer, U., Dabauvalle, M.-C., Merkert, H., and Benavente, R. (1988). The nuclear envelope and the organization of the pore complex. Cell Biol. Int. Rep. 12, 669-689.

Seelig, H.P., Schranz, P., Schroter, H., Wiemann, C., Griffiths, G., and Renz, M. (1994). Molecular genetic analyses of a 376-kilodalton Golgi complex membrane protein. Mol. Cell. Biol. 14, 2564-2576.

Shah, S., Tugendreich, S., and Forbes, D. (1998). Major binding sites for the nuclear import receptor are the internal nucleoporin Nup153 and the adjacent nuclear filament protein Tpr. J. Cell Biol. 141, 31-49.

Siegel, L.M., and Monty, K.J. (1966). Determination of molecular weights and frictional ratios of proteins in impure systems by use of gel filtration and density gradient centrifugation: application to crude preparations of sulfite and hydroxylamine reductases. Biochim. Biophys. Acta 112, 346-362.

Singer, R.H., and Green, M.R. (1997). Compartmentalization of eucaryotic gene expression: causes and effects. Cell 91, 291-294.

Sohda, M., Misumi, Y., Fujiwara, T., Nishioka, M., and Ikehara, Y. (1994). Molecular cloning and sequence analysis of a human 372kDa protein localized in the Golgi complex. Biochem. Biophys. Res. Commun. 205, 1399-1408.

Soman, N.R., Correa, P., Ruiz, B.A., and Wogan, G.N. (1991). The TPR-MET oncogenic rearrangement is present and expressed in human gastric carcinoma and precursor lesions. Proc. Natl. Acad. Sci. USA 88, 4892-4896.

Stoffler, D., Fahrenkrog, B., and Aebi, U. (1999). The nuclear pore complex: from molecular architecture to functional dynamics. Curr. Opin. Cell Biol. 11, 391-401.

Strambio-de-Castilia, C., Blobel, G., and Rout, M.P. (1999). Proteins connecting the nuclear pore complex with the nuclear interior. J. Cell Biol. 144, 839-855.

Sukegawa, J., and Blobel, G. (1993). A nuclear pore complex protein that contains zinc finger binding motifs, binds DNA, and faces the nucleoplasm. Cell 72, 29-38.

Takashima, T., Matsumura, S., Kariya, T., Sunaga, T., and Kuman, A. (1988). Studies on the physical states of human platelet myosin in crude extracts. J. Biochem. 104, 1027-1035.

Trybus, K.M., Huiatt, T.W., and Lowey, S. (1982). A bent monomeric conformation of myosin from smooth muscle. Proc. Natl. Acad. Sci. USA 79, 6151-6155.

Ye, Q., and Worman, H.J. (1995). Protein-protein interactions between human nuclear lamins expressed in yeast. Exp. Cell Res. 219, 292-298.

Yuan, L., Pelttari, J., Brundell, E., Björkroth, B., Zhao, J., Liu, J.-G., Brismar, H., Daneholt, B., and Höög, C. (1998). The synaptonemal complex protein SCP3 can form multistranded, cross-striated fibers in vivo. J. Cell Biol. 142, 331-339.

Zimowska, G., Aris, J.P., and Paddy, M.R. (1997). A Drosophila Tpr protein homolog is localized both in the extrachromosomal channel network and to nuclear pore complexes. J. Cell Sci. 110, 927-944. 